\title{
Modelling and parameter identification of electromechanical systems for energy harvesting and sensing
}

\author{
Adnan Kefal ${ }^{*^{1,2}}$, Claudio Maruccio* ${ }^{3}$, Giuseppe Quaranta ${ }^{4}$, and Erkan Oterkus ${ }^{5}$ \\ ${ }^{1}$ Faculty of Naval Architecture and Ocean Engineering, Istanbul Technical University, \\ Istanbul, Turkey, kefaladnan@itu.edu.tr \\ ${ }^{2}$ Integrated Manufacturing Technologies Research and Application Center, Sabanci \\ University, Istanbul, Turkey, adnankefal@sabanciuniv.edu \\ ${ }^{3}$ Department of Innovation Engineering, University of Salento, Lecce, Italy, \\ claudio.maruccio@unisalento.it \\ ${ }^{4}$ Department of Structural Engineering and Geotechnics, La Sapienza University, Rome, \\ Italy, giuseppe.quaranta@uniroma1.it \\ ${ }^{5}$ Department of Naval Architecture, Ocean and Marine Engineering, University of \\ Strathclyde, Glasgow, United Kingdom, erkan.oterkus@strath.ac.uk \\ *These authors contribute equally to this work
}

September 30, 2018

Keywords: Piezoelectric solid, Finite element method, state-space models, energy harvesting, parameter identification, Sensitivity analysis.

\begin{abstract}
Advanced modelling of electro-mechanical systems for energy harvesting (EH) and sensing is important to develop reliable self-powered autonomous electronic devices and for structural health monitoring (SHM). In this perspective, a novel computational approach is here proposed for both real-time and off-line parameter identification (PI). The system response is governed by a set of four partial differential equations (PDE) where the three displacement components and the electrical potential are the unknowns. Firstly, the finite element (FE) method is used to reduce the PDE problem into a set of ordinary differential equations (ODE). Then, a statespace model is derived with the aim to limit the PI problem to a subset of unknowns. After that, an identification error is introduced and the Lyapunov theory is used to derive the PI algorithm. The numerical implementation is based on a sensitivity analysis feedback block. The overall proposed computational strategy is robust and results in an exponential asymptotic convergence. The accuracy of the PI method is demonstrated by analysing the time-domain response of an array of piezoelectric bimorphs subjected to low-frequency structural random vibrations. The selected case-study is an existing cable-stayed bridge, for which an extensive dynamic monitoring campaign has provided the experimental data. Once time histories of the device response are obtained through time--dependent dynamic FE simulations, the PI algorithm is used to determine the unknown lumped coefficients of the state-space model. The comparison between FE method and lumped parameters model in terms of tip displacement and output voltage demonstrates the superior predictive capability of the new PI algorithm. As a result of the sensitivity analysis, guidelines to assess the optimal array configuration are also provided.
\end{abstract}




\section{Introduction}

Electromechanical systems are a fundamental component of a wide class of devices, such as actuators, sensors, controllers, motors and transducers. Several recent scientific researches about electromechanical systems are also focused on energy harvesting technologies with the aim to convert mechanical vibrations into electrical energy, thereby facilitating the development of small electronic autonomous apparatuses [20,29]. To achieve this result, several prototypes have been built in the last years based on thermoelectric, electromagnetic, pyroelectric, triboelectric and piezoelectric effects [44]. The length of these devices range from the nanoscale to the microscale, up to the macroscale. In this framework, advanced modelling and experimental characterization of the dynamic response are important to enhance the electromechanical systems performances. A reliable modelling is one of the main task in order to predict the response of complex intelligent materials and structures, given external loads and boundary conditions [13]. Furthermore, the continuous growth of the complexity in smart, micro and nano electromechanical devices calls for multiphysics and multiscale numerical simulations $[45,46,47]$. At the same time, experimental data are essential to assess the reliability of the model predictions. Given the model and the experimental data, dynamic system identification can be accomplished.

System identification [4] is the science to build a valid mathematical model capable of describing the essential properties of a given system starting from observed input-output or output-only data [12]. Despite the large amount of significant contributions about system identification [14, 15, 16, 17], some open issues still exist such as dealing with nonlinear models [11] and large databases (Bayesian and sensor networks [14]), formulating the estimation method as a convex optimization problem, bridging the gap between the scientific communities involved in model order reduction and system identification $[12,37,38]$. In particular, parameter estimation is the task that concerns with the experimental determination of the numerical values of the parameters governing the static and/or dynamic behaviour of a given system, under the assumption that the system model can be represented by means of a known mathematical structure [15]. Parameter estimation also plays an important role in filtering, state estimation [8] and controller design [9, 10, 11]. Parameter identification (PI) techniques can be classified into two main categories, namely parametric and non-parametric methods [19]. Unlike non-parametric methods (which are adopted when the system model structure is unknown), parametric methods rely on the availability of an accurate system model whose parameters have to be identified. Both frequency-domain (FD) [18] and time-domain (TD) approaches can be employed to this end.

Typical numerical identification approaches adopted in PI problems include Newton methods, least squares and the gradient search techniques [39, 40, 41] as well as soft computing techniques [42, 43]. For example, a generalized computing paradigm based on artificial dynamic models is proposed in [33], and the computational strategy therein proposed does not fail even when traditional iterative algorithms such as Newton-Raphson are not able to converge due to high nonlinearities. Among the different available approaches, the model reference adaptive systems (MRAS) technique can also be mentioned [50, 51]. It can be used for the direct adaptation of the controller gains $[52,53,54,55]$ as well as for the identification of the parameters of unknown processes [15, 31, 32, 34]. Recently, dynamic modeling of breast tissue with application of the MRAS identification technique based on clinical robot-assisted palpation is described in [5]. Gatto et al. [7] presented an online discretetime PI algorithm suitable for surface-mounted permanent magnet synchronous machines. Liu et al. [6] developed two MRAS estimators for identifying the parameters of permanent magnet synchronous motors based on the Lyapunov stability theorem and the Popov stability criterion, respectively. Lyapunov stability theory in analysis and control of electromechanical systems is discussed in [35, 27, 28]. 
During the last decade, even in the framework of smart electromechanical systems modelling [21, 22, 23, 24], several efforts have been spent in developing and implementing identification techniques based on parametric methods. Actually, the identification of electromechanical modal parameters of piezoelectric structures is important to enable a correct implementation of these devices for energy harvesting, vibration control and health monitoring applications [48]. Reduced-order modal models can usually arise from numerical finite element (FE) [49] or distributed analytical approaches $[21,23]$. Porfiri et al. [25] proposed two techniques for estimating piezoelectric modal couplings and piezoelectric modal capacitances. Erturk et al. [21] provided an alternative approach to conventional techniques for damping identification based on closed-form expressions and a single data point of the voltage frequency response function (FRF). Delpero et al. [26] reported that an accurate prediction of the damping is based on a reliable identification of the generalized coupling coefficient and provided experimental proof for shunted piezoelectric elements. A FD technique has been adopted in [24] in order to fit nonlinear algebraic equations derived via the method of harmonic balance.

In this study, we propose a novel computational approach for both real-time or off-line PI in the framework of electromechanical devices employed for energy harvesting and sensing applications [36]. The system response is governed by a set of four partial differential equations (PDE) where the three displacement components and the electrical potential are the unknowns. The FE method is first used to reduce the PDE problem into a set of ordinary differential equations (ODE). Then, a state-space model of the device response is derived with the aim to limit the PI problem to a subset of unknowns. Model order reduction is achieved since the resulting FE equations are projected onto the modal space. An identification error is then introduced and the Lyapunov theory is used to derive the PI algorithm. The underlying principle of the algorithm is to formulate an artificial dynamic system $[2,30]$ by a proper set of ODE, whose equilibrium points correspond to the problem solutions $[1,3]$. Exponential asymptotic convergence to equilibrium points for the artificial dynamic system is also achieved through a proper design, where a sensitivity analysis of the system response with respect to the vector of parameters to be identified is required.

\section{Modelling}

\subsection{PDE for a piezoelectric solid}

The governing equations for a linear piezoelectric solid are:

$$
\sigma_{i j, j}=\rho \frac{\partial^{2} u_{i}}{\partial t^{2}}, \quad \sigma_{i j}=\sigma_{j i}, \quad i \neq j, \quad \epsilon_{i j}=\frac{1}{2}\left(u_{i, j}+u_{j, i}\right), \quad D_{i, i}=\rho_{e}, \quad E_{i}=-\phi_{i},
$$

where stress/strain tensor and electric-field/electric-displacement vector components are defined by $\sigma_{i j}, \epsilon_{i j}, E_{i}$ and $D_{i}$, respectively. Moreover, $u_{i}$ are the displacement components $(i=1 . .3), \rho_{e}$ is the free electric charge density and $\phi$ is the electric potential. The electric entalpy $\mathcal{H}$ is defined in terms of strain and electric field vector components as [85]:

$$
\mathcal{H}=\frac{1}{2} C_{i j k l} \epsilon_{i j} \epsilon_{k l}-\frac{1}{2} \mu_{i j} E_{i} E_{j}-e_{k i j} \epsilon_{i j} E_{k},
$$


where $C_{i j k l}, e_{i k l}, \mu_{i k}$ are the elasticity, piezoelectric and dielectric material tensors. Consequently, the constitutive relations are:

$$
\begin{aligned}
\sigma_{i j} & =\frac{\partial \mathcal{H}}{\partial \epsilon_{i j}}=C_{i j k l} \epsilon_{k l}-e_{k i j} E_{k}, \\
D_{i} & =-\frac{\partial \mathcal{H}}{\partial E_{i}}=e_{i k l} \varepsilon_{k l}+\mu_{i k} E_{k} .
\end{aligned}
$$

The boundary conditions are defined as (see Figure 1):

$$
\begin{aligned}
& \mathbf{u}=\mathbf{0} \quad \text { on } \Gamma_{e}^{u_{1}}, \quad \mathbf{u}=\mathbf{u}_{0} \quad \text { on } \Gamma_{e}^{u_{2}}, \\
& \phi=0 \quad \text { on } \Gamma_{e}^{\phi_{1}}, \quad \phi=\phi_{0} \quad \text { on } \Gamma_{e}^{\phi_{2}}, \\
& \mathbf{n} \cdot \boldsymbol{\sigma}=\overline{\boldsymbol{\sigma}} \text { on } \Gamma_{n}^{\sigma}, \\
& \mathbf{n} \cdot \mathbf{D}=\overline{\mathbf{d}} \text { on } \Gamma_{n}^{D},
\end{aligned}
$$

where $\Gamma_{e}^{u_{1}}, \Gamma_{e}^{u_{2}}, \Gamma_{e}^{\phi_{1}}, \Gamma_{e}^{\phi_{2}}, \Gamma_{n}^{\sigma}, \Gamma_{n}^{D}$ are the domain boundaries for displacement, ground condition, electric potential, stress and electric displacement, respectively. The vector $\mathbf{n}$ is the normal to the domain boundary and $\mathbf{u}_{0}, \phi_{0}, \overline{\boldsymbol{\sigma}}, \overline{\mathbf{d}}$ are prescribed values of displacement, electric potential, stress and electric displacement. With some algebra, the final system of four coupled equations (strong form) for the three components of the displacement and the electric potential is obtained:

$$
C_{i j k l} \epsilon_{k l, j}+e_{k i j} \phi_{, k j}=\rho \frac{\partial^{2} u_{i}}{\partial t^{2}}, \quad e_{i k l} \epsilon_{k l, i}-\mu_{i k} \phi_{, i k}=\rho_{e}
$$

\subsection{Toward an ODE-based formulation}

Strain is obtained by derivatives of displacement, while displacement components and electric potential are defined in terms of shape functions $N^{a}$ and nodal point values $u_{i}^{a}$ and $\phi^{a}$ such as:

$$
\begin{gathered}
u_{i} \approx u_{i}^{h}=\sum_{a=1}^{n_{e}} N^{a} u_{i}^{a}, \\
\phi \approx \phi^{h}=\sum_{a=1}^{n_{e}} N^{a} \phi^{a},
\end{gathered}
$$

where $n_{e}$ denotes the number of nodes for each FE. Finally, the equivalent FE equations are written in matrix form as [82]:

$$
\left(\begin{array}{cc}
\mathbf{M}_{u u} & \mathbf{0} \\
\mathbf{0} & \mathbf{0}
\end{array}\right)\left(\begin{array}{c}
\ddot{\mathbf{u}}_{d} \\
\ddot{\boldsymbol{\phi}}_{d}
\end{array}\right)+\left(\begin{array}{cc}
\mathbf{C}_{u u} & \mathbf{0} \\
\mathbf{0} & \mathbf{0}
\end{array}\right)\left(\begin{array}{c}
\dot{\mathbf{u}}_{d} \\
\dot{\boldsymbol{\phi}}_{d}
\end{array}\right)+\left(\begin{array}{cc}
\mathbf{K}_{u u} & \mathbf{K}_{\mathrm{u} \phi} \\
\mathbf{K}_{\mathrm{u} \phi}^{T} & -\mathbf{K}_{\phi \phi}
\end{array}\right)\left(\begin{array}{c}
\mathbf{u}_{d} \\
\boldsymbol{\phi}_{d}
\end{array}\right)=\left(\begin{array}{c}
\mathbf{f} \\
\mathbf{f}_{e}
\end{array}\right)
$$

where $\mathbf{u}_{d}$ and $\boldsymbol{\phi}_{d}$ collect the value of the unknowns at the nodes of the FE discretization (the upper dot indicates the time derivatives) whereas $\mathbf{f}$ and $\mathbf{f}_{e}$ are the load vectors due to mechanical and electrical Neumann boundary conditions, respectively. Moreover, according to the standard FE procedure, the matrices in Eq. (8) are assembled as:

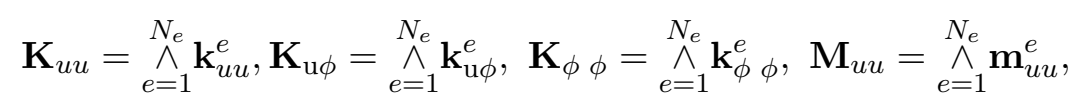


where:

$$
\begin{array}{r}
\mathbf{k}_{u u}^{e}=\int_{\Gamma_{e}}\left(\mathbf{B}^{u}\right)^{T} \mathbf{c B}^{u} d \Gamma, \\
\mathbf{k}_{\phi \phi}^{e}=\int_{\Gamma_{e}}\left(\mathbf{B}^{\phi}\right)^{T} \boldsymbol{\mu}^{T} \mathbf{B}^{\phi} d \Gamma, \\
\mathbf{k}_{\mathbf{u} \phi}^{e}=\int_{\Gamma_{e}}\left(\mathbf{B}^{u}\right)^{T} \mathbf{e}^{T} \mathbf{B}^{\phi} d \Gamma .
\end{array}
$$

The symbol $\wedge_{e=1}^{N_{e}}$ indicates the summation over the $N_{e}$ elements of the finite element mesh whereas the matrices $\mathbf{B}^{u}$, and $\mathbf{B}^{\phi}$ collect the derivatives of the shape functions. Moreover, $\mathbf{c}, \mathbf{e}$ and $\boldsymbol{\mu}$ are the elasticity, piezoelectric and permittivity matrices. Viscous damping is assumed in Eq. (8), where $\mathbf{C}_{u u}$ is the damping matrix. According to the Rayleigh damping model, $\mathbf{C}_{u u}$ is computed via the combination of the linear stiffness matrix and the mass matrix as $\mathbf{C}_{u u}=\alpha_{K} \mathbf{K}_{u u}+\alpha_{M} \mathbf{M}_{u u}$. The class of Newmark schemes and an implicit solver are used here for the time discretization $[56,57,84]$.

\subsection{Model order reduction}

In the previous section, the general electromechanical PDE are converted into a linear second order system with $N_{g}$ degrees of freedom based on the FE approximation. The differential equation of motion can be written in matrix form as:

$$
\mathbf{M}_{u u} \ddot{\mathbf{u}}_{d}+\mathbf{C}_{u u} \dot{\mathbf{u}}_{d}+\mathbf{K}_{u u} \mathbf{u}_{d}+\mathbf{K}_{u \phi} \phi_{d}=\mathbf{f} .
$$

For the class of problems of interest in this paper, the mechanical Neumann boundary conditions are given by:

$$
\mathbf{f}=-\mathbf{M}_{u u} \mathbf{S}_{g} \ddot{u}_{g}(t),
$$

where $\mathbf{f}$ is an effective force vector due to the external vibrations, $\ddot{u}_{g}$ is the horizontal base acceleration, $\mathbf{S}_{g}$ is an influence vector whose components that refer to the motion direction are equal to 1, see [58]. In practice, $-\mathbf{M}_{u u} \mathbf{S}_{g}$ describes the spatial distribution of the effective force and $\ddot{u}_{g}(t)$ is their time variation. The following coordinate transformation is used for the mechanical and electrical degrees of freedom:

$$
\mathbf{u}_{d}=\boldsymbol{\Phi} \boldsymbol{\eta}, \quad \phi_{d}=\boldsymbol{\Phi} \mathbf{v}
$$

thereby allowing the projection of the dynamic equations onto the modal space, where $\boldsymbol{\Phi}=$ $\left[\boldsymbol{\Phi}_{1}, \ldots, \boldsymbol{\Phi}_{N}\right]$ is the modal matrix, $\boldsymbol{\eta}=\left[\eta_{1}, \ldots, \eta_{N}\right]^{T}$ is the vector of modal mechanical coordinates, and $\mathbf{v}=\left[v_{1}, \ldots, v_{N}\right]^{T}$ is the vector of modal electrical coordinates.

Once Eq. (15) is substituted into Eq. (13), both sides of Eq. (13) are pre-multiplied by $\boldsymbol{\Phi}^{T}$ and post-multiplied by $\boldsymbol{\Phi}$, thus obtaining:

$$
\boldsymbol{\Phi}^{T} \mathbf{M}_{u u} \boldsymbol{\Phi} \ddot{\boldsymbol{\eta}}_{d}+\boldsymbol{\Phi}^{T} \mathbf{C}_{u u} \boldsymbol{\Phi} \dot{\boldsymbol{\eta}}_{d}+\boldsymbol{\Phi}^{T} \mathbf{K}_{u u} \boldsymbol{\Phi} \boldsymbol{\eta}_{d}+\boldsymbol{\Phi}^{T} \mathbf{K}_{u \phi} \boldsymbol{\Phi} \mathbf{v}=-\boldsymbol{\Phi}^{T} \mathbf{M}_{u u} \mathbf{S}_{g} \ddot{u}_{g}(t)=-\boldsymbol{\Gamma} \boldsymbol{\Phi}^{T} \mathbf{M}_{u u} \boldsymbol{\Phi} \ddot{u}_{g}(t),
$$

where $\boldsymbol{\Gamma}=\left(\boldsymbol{\Phi}^{T} \mathbf{M}_{u u} \mathbf{S}_{g}\right) /\left(\boldsymbol{\Phi}^{T} \mathbf{M}_{u u} \boldsymbol{\Phi}\right)$ is the modal participation vector. In particular, it yields that $\Gamma_{r}=\left(\boldsymbol{\Phi}_{r}^{T} \mathbf{M}_{u u} \mathbf{S}_{g}\right) /\left(\boldsymbol{\Phi}_{r}^{T} \mathbf{M}_{u u} \boldsymbol{\Phi}_{r}\right)$, with $r=1 \ldots N$. Modal shapes $\boldsymbol{\Phi}_{r}$ are determined by solving the eigenproblem:

$$
\mathbf{K}_{u u}^{e f f} \boldsymbol{\Phi}_{r}=\omega_{r}^{2} \mathbf{M}_{u u} \boldsymbol{\Phi}_{r}
$$


where

$$
\mathbf{K}_{u u}^{e f f}=\left(\mathbf{K}_{u u}-\mathbf{K}_{u \phi} \mathbf{K}_{\phi \phi}^{-1} \mathbf{K}_{u \phi}^{T}\right),
$$

and $\omega_{r}^{2}$ are the corresponding eigenvalues. If we define the modal mass, damping, stiffness and piezoelectric matrices as:

$$
\tilde{\mathbf{M}}_{u u}=\boldsymbol{\Phi}^{T} \mathbf{M}_{u u} \boldsymbol{\Phi}, \quad \tilde{\mathbf{C}}_{u u}=\boldsymbol{\Phi}^{T} \mathbf{C}_{u u} \boldsymbol{\Phi}, \quad \tilde{\mathbf{K}}_{u u}=\boldsymbol{\Phi}^{T} \mathbf{K}_{u u} \boldsymbol{\Phi}, \quad \tilde{\boldsymbol{\Theta}}_{q}=\boldsymbol{\Phi}^{T} \mathbf{K}_{u \phi} \boldsymbol{\Phi},
$$

respectively, the resulting system of equations is decoupled and can be written as:

$$
\tilde{\mathbf{M}}_{u u} \ddot{\boldsymbol{\eta}}+\tilde{\mathbf{C}}_{u u} \dot{\boldsymbol{\eta}}+\tilde{\mathbf{K}}_{u u} \boldsymbol{\eta}=-\left(\boldsymbol{\Gamma} \tilde{\mathbf{M}}_{u u} \ddot{u}_{g}(t)+\tilde{\boldsymbol{\Theta}}_{q} \mathbf{v}\right) .
$$

Similarly, once the time derivative of the electrical equation is performed, its projection onto the modal space is obtained as follows:

$$
\boldsymbol{\Phi}^{T} \mathbf{K}_{\phi u} \boldsymbol{\Phi} \dot{\eta}-\boldsymbol{\Phi}^{T} \mathbf{K}_{\phi \phi} \boldsymbol{\Phi} \dot{\mathbf{v}}=\boldsymbol{\Phi}^{T} \dot{\mathbf{Q}}=\boldsymbol{\Phi}^{T} \mathbf{R}_{L}^{-1} \boldsymbol{\Phi} \mathbf{v}
$$

where $\mathbf{R}_{L}$ is the vector of nodal impedances and $\dot{\mathbf{Q}}$ indicates electrical currents.

Now, we define modal resistance and capacitance matrices as:

$$
\tilde{\mathbf{R}}_{L}=\boldsymbol{\Phi}^{T} \mathbf{R}_{L}^{-1} \boldsymbol{\Phi}, \quad \tilde{\mathbf{C}}_{p}=\boldsymbol{\Phi}^{T} \mathbf{K}_{\phi \phi} \boldsymbol{\Phi},
$$

respectively. Therefore, Eq. (21) becomes:

$$
\tilde{\boldsymbol{\Theta}}_{q}^{T} \dot{\boldsymbol{\eta}}-\tilde{\mathbf{C}}_{p} \dot{\mathbf{v}}=\tilde{\mathbf{R}}_{L}^{-1} \mathbf{v} .
$$

\subsection{Derivation of the state-space model}

Equations (20) and (23) can be arranged in the state-space form as:

$$
\left(\begin{array}{c}
\dot{\boldsymbol{\eta}}(t) \\
\ddot{\boldsymbol{\eta}}(t) \\
\dot{\mathbf{v}}(t)
\end{array}\right)=\left(\begin{array}{ccc}
\mathbf{0} & \mathbf{I} & \mathbf{0} \\
-\tilde{\mathbf{K}}_{u u} \tilde{\mathbf{M}}_{u u}^{-1} & -\tilde{\mathbf{C}}_{u u} \tilde{\mathbf{M}}_{u u}^{-1} & -\tilde{\boldsymbol{\Theta}}_{q} \tilde{\mathbf{M}}_{u u}^{-1} \\
\mathbf{0} & \tilde{\boldsymbol{\Theta}}_{q}^{T} \tilde{\mathbf{C}}_{p}^{-1} & -\left(\tilde{\mathbf{R}}_{L} \tilde{\mathbf{C}}_{p}\right)^{-1}
\end{array}\right)\left(\begin{array}{l}
\boldsymbol{\eta}(t) \\
\dot{\boldsymbol{\eta}}(t) \\
\mathbf{v}(t)
\end{array}\right)+\left(\begin{array}{c}
\mathbf{0} \\
-\boldsymbol{\Gamma} \ddot{u}_{g}(t) \\
\mathbf{0}
\end{array}\right),
$$

where we can introduce the matrices $\Omega^{2}, \Lambda$ and $\Theta$ :

$$
\boldsymbol{\Omega}^{2}=\tilde{\mathbf{K}}_{u u} \tilde{\mathbf{M}}_{u u}^{-1}, \quad \boldsymbol{\Lambda}=\tilde{\mathbf{C}}_{u u} \tilde{\mathbf{M}}_{u u}^{-1}, \quad \boldsymbol{\Theta}=\tilde{\boldsymbol{\Theta}}_{q} \tilde{\mathbf{M}}_{u u}^{-1}
$$

and the vector $\tilde{\mathbf{f}}$ such as:

$$
\tilde{\mathbf{f}}=-\Gamma \ddot{u}_{g}(t) .
$$

Moreover, the orthogonality condition leads to the following diagonal matrices:

$$
\tilde{\mathbf{M}}_{u u}=\left(\begin{array}{ccc}
M_{1} & & \\
& \ddots & \\
& & M_{N}
\end{array}\right), \quad \boldsymbol{\Omega}^{2}=\left(\begin{array}{ccc}
\omega_{1}^{2} & & \\
& \ddots & \\
& & \omega_{N}^{2}
\end{array}\right), \quad \boldsymbol{\Lambda}=\left(\begin{array}{ccc}
2 \zeta_{1} \omega_{1} & & \\
& \ddots & \\
& & 2 \zeta_{N} \omega_{N}
\end{array}\right),
$$




$$
\begin{gathered}
\tilde{\mathbf{R}}_{L}=\left(\begin{array}{ccc}
R_{L, 1} & & \\
& \ddots & \\
& & R_{L, N}
\end{array}\right), \quad \tilde{\mathbf{C}}_{p}=\left(\begin{array}{ccc}
C_{p, 1} & & \\
& \ddots & \\
& & C_{p, N}
\end{array}\right), \quad \boldsymbol{\Theta}=\left(\begin{array}{ccc}
\Theta_{1} / M_{1} & & \\
& \ddots & \\
& & \Theta_{N} / M_{N}
\end{array}\right), \\
\tilde{\mathbf{f}}=\left(\begin{array}{c}
f_{1} \\
\vdots \\
f_{N}
\end{array}\right)=-\left(\begin{array}{c}
\Gamma_{1} \ddot{u}_{g}(t) \\
\vdots \\
\Gamma_{N} \ddot{u}_{g}(t)
\end{array}\right) .
\end{gathered}
$$

\section{Identification algorithm based on sensitivity analysis}

The system given by Eq. (24) can be rewritten as follows:

$$
\dot{\mathbf{z}}=\mathbf{A z}+\mathbf{B u},
$$

where

$$
\mathbf{z}=[\boldsymbol{\eta}, \dot{\boldsymbol{\eta}}, \mathbf{v}]^{T}, \quad \mathbf{u}=[0, \tilde{\mathbf{f}}, 0]^{T}, \quad \mathbf{B}=[\mathbf{0}, \mathbf{I}, \mathbf{0}]^{T},
$$

and

$$
\mathbf{A}=\left(\begin{array}{ccc}
\mathbf{0} & \mathbf{I} & \mathbf{0} \\
-\boldsymbol{\Omega}^{2} & -\boldsymbol{\Lambda} & -\boldsymbol{\Theta} \\
\mathbf{0} & \boldsymbol{\Theta} \tilde{\mathbf{C}}_{p}^{-1} & -\left(\tilde{\mathbf{R}}_{L} \tilde{\mathbf{C}}_{p}\right)^{-1}
\end{array}\right)
$$

The matrix $\mathbf{A}$ represents the state matrix whereas the matrix $\mathbf{B}$ is the input matrix. Moreover, we can introduce an output vector:

$$
\mathbf{y}=\mathbf{C z}+\mathbf{D u}
$$

where, for the electromechanical system under consideration, $\mathbf{D}=\mathbf{0}$ and $\mathbf{C}=\left(\begin{array}{lll}\mathbf{I} & \mathbf{0} & \mathbf{0} \\ \mathbf{0} & \mathbf{0} & \mathbf{I}\end{array}\right)$. Overall, the set of equations:

$$
\left\{\begin{array}{l}
\dot{\mathbf{z}}=\mathbf{A z}+\mathbf{B u} \\
\mathbf{y}=\mathbf{C z}+\mathbf{D u}
\end{array}\right.
$$

fully describes the linear time-invariant electromechanical system.

Now, let $\boldsymbol{\lambda}$ be a vector of unknown parameters that characterize the dynamic response of the electromechanical system described by Eq. (24):

$$
\boldsymbol{\lambda}=\left[\lambda_{1}, \lambda_{2}, \ldots, \lambda_{S}\right]^{T},
$$

where $S$ is the number of parameters to be identified in the matrix $\mathbf{A}$. Our goal is to find the set $\lambda^{*}$ that minimizes the difference:

$$
\mathbf{e}(\boldsymbol{\lambda}, t)=\mathbf{y}(\boldsymbol{\lambda}, t)-\mathbf{y}_{m}(t),
$$

where the vector $\mathbf{y}_{m}(t)$ includes the values of measured displacements and electrical potentials of the electromechanical system whose parameters we want to identify. The simplest approach to solve this problem is to minimize the sum of the squared residuals. Therefore, the parametric 
identification process can be formulated as the following minimization problem:

$$
\left\{\begin{array}{l}
\min _{\boldsymbol{\lambda}} \quad \psi(\boldsymbol{\lambda}, t) \quad \forall t \\
\psi(\boldsymbol{\lambda}, t)=\frac{1}{2} \sum_{\iota=1}^{P_{m}} e_{\iota}(\boldsymbol{\lambda}, t)^{2}=\frac{1}{2} \mathbf{e}(\boldsymbol{\lambda}, t)^{T} \mathbf{e}(\boldsymbol{\lambda}, t)
\end{array},\right.
$$

where $P_{m}$ is the number of control/measurement points and $\iota=1 \ldots P_{m}$. Following [33], we now assume that $\dot{\boldsymbol{\lambda}}(t)$ changes according to the gradient of $\psi$, that is:

$$
\dot{\boldsymbol{\lambda}}(t)=-\boldsymbol{\kappa}\left(\frac{\mathcal{D} \psi(\boldsymbol{\lambda}, t)}{\mathcal{D} \boldsymbol{\lambda}(t)}\right)^{T}=-\boldsymbol{\kappa}\left(\frac{\mathcal{D} \mathbf{e}(\boldsymbol{\lambda}, t)}{\mathcal{D} \boldsymbol{\lambda}(t)}\right)^{T} \mathbf{e}(\boldsymbol{\lambda}, t),
$$

where $\boldsymbol{\kappa}$ is a diagonal matrix and $\mathcal{D}$ is the directional derivative. Hence, we obtain:

$$
\dot{\psi}(\boldsymbol{\lambda}, t)=-\boldsymbol{\kappa}\left[\mathbf{e}(\boldsymbol{\lambda}, t)^{T} \frac{\mathcal{D} \mathbf{e}(\boldsymbol{\lambda}, t)}{\mathcal{D} \boldsymbol{\lambda}(t)}\left(\frac{\mathcal{D} \mathbf{e}(\boldsymbol{\lambda}, t)}{\mathcal{D} \boldsymbol{\lambda}(t)}\right)^{T} \mathbf{e}(\boldsymbol{\lambda}, t)\right] .
$$

Moreover, observing that $\psi$ is positive-semidefinite and his time derivative $\dot{\psi}$ negative-semidefinite, the Lyapunov theorem guarantees that if the vector $\boldsymbol{\lambda}(t)$ evolves according to Eq. (38), it converges to an equilibrium point $\boldsymbol{\lambda}^{*}$ which is asymptotically stable and represents the solution of the identification problem. However, the directional derivative of the error $\mathbf{e}(\boldsymbol{\lambda}, t)$ with respect to $\boldsymbol{\lambda}$ must be computed to solve Eq. (38). It is defined as follows:

$$
\frac{\mathcal{D} \mathbf{e}(\boldsymbol{\lambda}, t)}{\mathcal{D} \boldsymbol{\lambda}}=\frac{\mathcal{D}\left[\mathbf{y}(\boldsymbol{\lambda}, t)-\mathbf{y}_{m}(t)\right]}{\mathcal{D} \boldsymbol{\lambda}}=\frac{\mathcal{D} \mathbf{y}(\boldsymbol{\lambda}, t)}{\mathcal{D} \boldsymbol{\lambda}}=\mathbf{C} \frac{\mathcal{D}[\mathbf{z}(\boldsymbol{\lambda}, t)]}{\mathcal{D} \boldsymbol{\lambda}}
$$

and it is strictly connected with the directional derivative of the system dynamic response $\mathbf{z}(\boldsymbol{\lambda}, t)$ with respect to $\boldsymbol{\lambda}$. Unfortunately, an implicit dependence exists between $\mathbf{z}(\boldsymbol{\lambda}, t)$ and $\boldsymbol{\lambda}$, where the exception is represented by the existence of simplified analytical solutions for $\mathbf{z}(\boldsymbol{\lambda}, t)$ in presence of particular input excitation $\mathbf{u}(t)$ (for example, if an harmonic force is applied, then the relation becomes explicit). However, for a general case, the dependence of the state-space system solution with respect to each unknown parameter must be considered and can be computed deriving Eq. (30) using the chain rule:

$$
\frac{\partial \dot{\mathbf{z}}(\boldsymbol{\lambda}, t)}{\partial \lambda_{s}}=\frac{\partial \mathbf{A}(\boldsymbol{\lambda}, t)}{\partial \lambda_{s}} \mathbf{z}(\boldsymbol{\lambda}, t)+\mathbf{A}(\boldsymbol{\lambda}, t) \frac{\partial \mathbf{z}(\boldsymbol{\lambda}, t)}{\partial \lambda_{s}} .
$$

It can be observed that Eq. (41) has the same form of Eq. (30). In fact, if we now introduce $\mathbb{Q}=\frac{\mathcal{D} \mathbf{z}(\boldsymbol{\lambda}, t)}{\mathcal{D} \boldsymbol{\lambda}}$ and $\mathbb{A}=\frac{\mathcal{D} \mathbf{A}(\boldsymbol{\lambda}, t)}{\mathcal{D} \boldsymbol{\lambda}}$, the set of $S$ equations given by Eq. (41) is recast in the form:

$$
\dot{\mathbb{Q}}=\mathbf{A} \mathbb{Q}+\mathbb{A} \mathbf{z}
$$

It is here noticed that $\dot{\mathbb{Q}}$ and $\mathbb{Q}$ are second order tensors, while $\mathbb{A}$ and $\mathbf{z}$ are third and first order tensors, respectively. Furthermore, if the uncertainty in the parameters is high, the convergence of the procedure can be enhanced using in the sensitivity dynamic equation (42), in place of the estimate of the state $\mathbf{z}(\boldsymbol{\lambda}, t)$ directly the experimental values.

By combining Eq. (42), Eq. (38) and Eq. (34), the identification problem can be solved looking for the solution of the following set of equations: 


$$
\left\{\begin{array}{l}
\dot{\mathbf{z}}(\boldsymbol{\lambda}(t), t)=\mathbf{A}(\boldsymbol{\lambda}(t), t) \mathbf{z}(\boldsymbol{\lambda}(t), t)+\mathbf{B u}(t) ; \\
\mathbf{y}(\boldsymbol{\lambda}(t), t)=\mathbf{C} \mathbf{z}(\boldsymbol{\lambda}(t), t)+\mathbf{D u}(t) ; \\
\dot{\mathbb{Q}}(\boldsymbol{\lambda}(t), t)=\mathbf{A}(\boldsymbol{\lambda}(t), t) \mathbb{Q}(\boldsymbol{\lambda}(t), t)+\mathbb{A}(\boldsymbol{\lambda}(t), t) \mathbf{z}(\boldsymbol{\lambda}(t), t) ; \\
\mathbb{Q}(\boldsymbol{\lambda}(t), t)=\frac{\mathcal{D} \mathbf{z}(\boldsymbol{\lambda}(t), t)}{\mathcal{D} \boldsymbol{\lambda}(t)} ; \\
\dot{\boldsymbol{\lambda}}(t)=-\boldsymbol{\kappa}\left(\mathbf{C} \frac{\mathcal{D}[\mathbf{z}(\boldsymbol{\lambda}(t), t)]}{\mathcal{D} \boldsymbol{\lambda}(t)}\right)^{T} \mathbf{e}(\boldsymbol{\lambda}(t), t) ; \\
\mathbf{e}(\boldsymbol{\lambda}(t), t)=\mathbf{y}(\boldsymbol{\lambda}(t), t)-\mathbf{y}_{m}(t) .
\end{array}\right.
$$

A block diagram of the proposed algorithm is provided in Figure 2.

\section{Benchmark of the method}

The configuration considered for the device under investigation is of the cantilevered type and the assumed model relies on the Euler-Beroulli beam theory [20, 23]. Piezoelectric bimorphs mounted as cantilever beam are widely used for energy harvesting applications. A common layout for piezoelectric bimorph consists of two layers joined together with different polarization. The top surface of the beam has an electrode patch, whereas the bottom surface is grounded. The piezoelectric bimorph is made of Polyvinylidene Fluoride (PVDF), whose properties are given in Table 1.

Table 1: Elastic, piezoelectric, and permittivity properties of PVDF.

\begin{tabular}{ll}
\hline Property & Value \\
\hline Young's modulus, $E_{1}$ & $2 \mathrm{GPa}$ \\
Poisson's ratio, $\nu_{12}$ & 0.29 \\
Shear modulus, $G_{12}$ & $0.775 \mathrm{GPa}$ \\
Piezoelectric strain coefficients, $d_{31}$ & $2.2 \times 10^{-11} \mathrm{C} / \mathrm{N}$ \\
Piezoelectric strain coefficients, $d_{32}$ & $0.3 \times 10^{-11} \mathrm{C} / \mathrm{N}$ \\
Piezoelectric strain coefficients, $d_{33}$ & $-3.0 \times 10^{-11} \mathrm{C} / \mathrm{N}$ \\
Relative permittivity at constant stress, $\mu_{33}$ & 12 \\
\hline
\end{tabular}

A reference bimorph geometry is now introduced, with length $L=30 \mathrm{~mm}$, width $B=10 \mathrm{~mm}$, thickness $H_{b}=0.2 \mathrm{~mm}$ and tip mass $M=25 \mathrm{~g}$. Close to the resonance, the system response given by Eq. (24) is well approximated using its first mode only. Therefore, in order to illustrate the effectiveness of the proposed computing paradigm, we considered here the final single-degree-offreedom (SDOF) electromechanical model resulting from the above assumption. As a consequence, for the device depicted in Figure 3 the electromechanical response is governed by the equations:

$$
\left\{\begin{array}{l}
\ddot{\eta}_{1}+2 \zeta_{1} \omega_{1} \dot{\eta}_{1}+\omega_{1}^{2} \eta_{1}+\theta_{1} v=F_{1}, \\
C_{1} \dot{v}+\frac{v}{R_{1}}-\theta_{1} \dot{\eta}_{1}=0,
\end{array}\right.
$$

where $\zeta_{1}$ is the first modal mechanical damping ratio, $\omega_{1}$ is the first undamped natural frequency, $\theta_{1}$ is the modal electromechanical coupling terms, $F_{1}$ is the first modal mechanical forcing function, $C_{1}$ is the capacitance, $R_{1}$ is the load resistance and $v=\left(V_{t}-V_{b}\right)$ is the voltage response across the external resistive load. For further information on the device material properties and geometry, the interested reader can refer to [49]. In practice, this is a single input $\left(F_{1}\right)$ and multiple output ( $\eta_{1}$ and $v$ ) system. In place of the experimental data, we use a predefined set of target parameters to benchmark the proposed method. The obtained trajectories of the relative error $\mathbf{e}(\boldsymbol{\lambda}, t)$ components 
Table 2: Acceleration data $\left[a_{g}\right]$ (time domain).

\begin{tabular}{llll}
\hline & Max & Min & RMS \\
S1 & 0.14 & -0.15 & 0.019 \\
S2 & 0.097 & -0.08 & 0.024 \\
S3 & 0.29 & -0.25 & 0.042 \\
S4 & 0.42 & -0.37 & 0.063 \\
S5 & 1.13 & -0.78 & 0.093 \\
\hline
\end{tabular}

(in terms of displacements and voltages) are provided in Figure 4 and Figure 5. Similarly, the dynamic evolution of the unknown parameters $K_{1}=M \omega_{1}^{2}$ and $\theta_{1}$ is depicted in Figure 6 and Figure 7. It is worth noting that the system converges to the target values after a time of 6 seconds. This time is function of the employed gain coefficients in the matrix $\boldsymbol{\kappa}$. The final values obtained are: $K_{1}=1.537 \mathrm{~N} / \mathrm{m}$ and $\theta_{1}=6.968 \times 10^{-6} \mathrm{C} / \mathrm{m}$.

This benchmark problem demonstrated that the proposed identification algorithm can identify the unknown system parameters and is able to follow the time evolution of the unknown dynamic system.

\section{Case-study}

\subsection{Experimental response of a cable-stayed bridge}

The selected case-study is an existing cable-stayed bridge that crosses the Garigliano river (Italy). An overview of the bridge is given in Figure 8. The bridge was built in 1993 and consists of two equal spans whose length is $90 \mathrm{~m}$. The width of the deck is $26.1 \mathrm{~m}$ and its height is $2.45 \mathrm{~m}$. The girder is constrained at the central tower, the height of which is $10.85 \mathrm{~m}$ from the foundation to the deck extrados and $30 \mathrm{~m}$ from the deck to the top. The girder is also simply supported at the other ends and sustained by 18 couples of cables (nine couples for each span). The cross sectional areas of the two shortest cables is $67.5 \mathrm{~cm}^{2}$. The longest cable has a cross sectional area of 70.5 $\mathrm{cm}^{2}$. The others have a cross sectional area equal to $82.5 \mathrm{~cm}^{2}$.

The dynamic response of the bridge under ambient vibrations was recorded by means of an extensive monitoring campaign [86]. The installed network of sensors consisted of piezoelectric uniaxial accelerometers PCB 393B12 with a sensitivity equal to $10 \mathrm{~V} / \mathrm{g}$ and a frequency range 0.15 $\mathrm{Hz}-1000 \mathrm{~Hz}$. The sampling rate was equal to $200 \mathrm{~Hz}$. The vertical vibrations of the deck were recorded by installing the accelerometers within the structure along the longitudinal axis. Both horizontal and vertical accelerations of each cable were monitored by placing two accelerometers at an average height of $3.8 \mathrm{~m}$ above the deck extrados. The intensity of the dynamic response of the cables was found larger than that of the deck. Therefore, only the vertical accelerations recorded on some cables are used in the present work. The time window of the measured cable response is about $50 \mathrm{~s}$. From the overall database, only five measurement points are selected and used for the following analyses. In Figure 8, these points are indicated as S1, S2, S3, S4 and S5.

The acceleration records of each sensor are provided in Figure 9 and Figure 10 in the time domain and frequency domain. The maximum value corresponds to 1.13 times the gravitational acceleration $\left(a_{g}=9.8 \mathrm{~m} / \mathrm{s}^{2}\right)$ and it occurs for $\mathrm{S} 5$ while the maximum root mean square (RMS) is found to be $0.093 a_{g}$, see Table 2 and Table 3 . 
Table 3: Acceleration data $\left[a_{g}\right]$ (frequency domain).

\begin{tabular}{lll}
\hline & $f_{c}[\mathrm{~Hz}]$ & Acceleration \\
S1 & 1.28 & $6.59 \times 10^{-4}$ \\
S2 & 3.35 & $1.23 \times 10^{-3}$ \\
S3 & 2.53 & $1.46 \times 10^{-3}$ \\
S4 & 3.22 & $1.90 \times 10^{-3}$ \\
S5 & 12.59 & $3.06 \times 10^{-3}$ \\
\hline
\end{tabular}

\subsection{Energy harvesting}

\subsubsection{Array of bimorphs}

Energy harvesting is the process of (1) capturing a non-electric form of energy from naturallyoccurring energy sources, (2) converting it into an electric energy, and (3) accumulating this electric energy for future uses [73]. Examples of energy sources typically exploited for energy harvesting applications include thermal, light (solar), wind, mechanical (vibration) and many others. Amongst these scavenging techniques, harnessing energy from vibration is probably the most promising approach to power wireless sensing electronics [69]. Current proposals for vibration-to-electricity conversion are mostly based on electrostatic $[71,76]$, electromagnetic $[64,68,78]$ or piezoelectric methods $[62,63,66,74]$. Within this framework, piezoelectric energy harvesting technologies are widely used [67] since the manufacturing processes of piezoelectric films are nowadays mature for large-scale applications.

To date, most piezoelectric vibration-based energy harvesting (EH) devices are designed as linear resonators that work efficiently within a limited bandwidth near the targeted resonant frequency [90]. If the identified excitation frequency slightly shifts with respect to the resonant frequency of the harvester, then its performance can decrease drastically [91, 92, 93, 94]. Many factors can jeopardize the optimal tuning. As a matter of fact, the frequency content of the dynamic input is unfortunately not known exactly in many cases. Moreover, the frequency content of many mechanical vibrations can vary in time. Furthermore, the input signal energy can be distributed over a quite large frequency range. Hence, increasing the bandwidth of piezoelectric vibrationbased EH devices is a critical design issue to foster their extensive use [59, 60, 65, 72]. The existing strategies to broaden the bandwidth of EH devices can be grouped into frequency tuning, multimodal energy harvesting and nonlinear energy harvesting [89]. Resonance tuning methods can be further categorized into mechanical, magnetic, and piezoelectric methods [61, 70, 75, 77, 79, 80]. The use of an array of piezoelectric EHs is among the simplest strategies, but its efficient design requires a proper evaluation of the role of mechanical and electrical parameters.

The main advantage of this technique is that the setup is scalable, since it is very easy to add or remove a single cantilever or to adjust their resonance frequencies. Nevertheless, this setup may require a bulky volume causing a limitation for small-scale devices. Therefore, designing an optimal bimorph geometry as well as assessing the optimal number of bimorphs is not a simple task.

It can be seen from the experimental measurements (Figure 9 and Figure 10) that the largest bridge cables vibrations are concentrated in the frequency range $0-12 \mathrm{~Hz}$. A first estimation of the maximum energy that can be extracted from a given base motion is possible based on a deterministic design of the generator as discussed in [81]. Following this approach, a parametric analysis has been performed for a preliminary design of each bimorph in the array. Based on such results, a tuning between the bimorph first natural frequency $f_{1}$ and the cable frequency $f_{c}$ (see Table 3 ) with the peak amplitude in the signal spectrum is achieved by selecting a proper thickness of the 
Table 4: Natural frequency of the bimorph.

\begin{tabular}{llll}
\hline Acceleration & $f_{c}$ & $H_{o}$ & $f_{1}\left(H=H_{o}\right)$ \\
data & {$[\mathrm{Hz}]$} & {$[\mathrm{mm}]$} & {$[\mathrm{Hz}]$} \\
S1 & 1.28 & 0.20 & 1.22 \\
S2 & 3.35 & 0.38 & 3.21 \\
S3 & 2.53 & 0.31 & 2.42 \\
S4 & 3.22 & 0.37 & 3.08 \\
S5 & 12.59 & 0.92 & 12.04 \\
\hline
\end{tabular}

cantilever.

\subsubsection{Parameter identification}

By tuning $f_{c}$ and $f_{1}$ for a given signal spectrum (from $\mathrm{S} 1$ to $\mathrm{S} 5$ ), the optimal bimorph thickness $H_{o}$ is calculated. The value is obtained such as $\left\|\left(f_{c}-f_{1}\right) / f_{1}\right\| \approx 5 \%$, see Table 4 . The shift is introduced in order to obtain a conservative estimation of the energy as well as to prevent the occurrence of high stress levels that would not be compatible with the material strength of the device. For the sake of completeness, it is remarked that the tuning condition can be achieved by designing a feedback controller. Also a change in the electrical boundary conditions (resistance/capacitance) can lead to a bandwidth shift. However, an electronic network (such as rectifier bridges and switches in the circuit) is required for this purpose and consequently both the size and cost of the system would increase [88].

First, FE analyses are implemented by discretizing the device geometry with eight-node coupledfield electromechanical elements. A simple circuit element is introduced between the two layers of each bimorph to simulate the electrical boundary conditions. A mass element is used to model the tip mass while the mechanical boundary conditions (i.e. random accelerations) are globally applied to the model at each time step. Then, the array parameters are identified. Third, the resulting set of ordinary differential equations with updated lumped coefficients is solved using a Runge-Kutta algorithm. According to [87], the assumption of linear piezoelectric material behaviour in EH made of PVDF layers is valid if the tip displacement is not larger than $0.25 L$, as in this case-study. Finally, the time histories of tip displacement and output voltage are obtained, see Figure 11 and Figure 12. The comparison between FE model and lumped parameters model demonstrates the effectiveness of the proposed algorithm.

\subsection{Sensing}

Although reduced order methods neglect local information about stress, strain and electric fields, they can be useful to achieve a compromise between accuracy and computational cost [83]. In the following numerical analyses, we analyze the bimorphs as a sensing apparatus and assess the influence on the response as function of geometrical and electrical variables. Hereafter, FE solutions only are shown for the sake of readability, but lumped parameters models well approximate these results.

\subsubsection{Influence of the geometry}

A comparison of the bimorph voltage response is provided for two different thicknesses, namely $H_{b}$ (red line) and $H_{o}$ (black line), see Figure 13. From Figure 13 and Table 5, it is evident that no difference is obtained for $\mathrm{S} 1$ since $H_{b}=H_{o}$ for this case. For a damping value equal to $8 \%$ and for 
Table 5: Bimorph output voltage [V] for different input acceleration (Max/Min).

\begin{tabular}{lllllll}
\hline & $H_{o}, \zeta=0 \%$ & $H_{o}, \zeta=4 \%$ & $H_{o}, \zeta=8 \%$ & $H_{b}, \zeta=0 \%$ & $H_{b}, \zeta=4 \%$ & $H_{b}, \zeta=8 \%$ \\
S1 & 42 & 17 & 10 & 42 & 17 & 10 \\
S2 & 65 & 26 & 15 & 4.46 & 3.67 & 3.20 \\
S3 & 51 & 19 & 12.7 & 20 & 14 & 10 \\
S4 & 106 & 34 & 22 & 19 & 7 & 5 \\
S5 & 107 & 45 & 29 & 3 & 0.90 & 0.65 \\
\hline
\end{tabular}

Table 6: Bimorph output voltage [V] for different input acceleration (RMS).

\begin{tabular}{lllllll}
\hline & $H_{o}, \zeta=0 \%$ & $H_{o}, \zeta=4 \%$ & $H_{o}, \zeta=8 \%$ & $H_{b}, \zeta=0 \%$ & $H_{b}, \zeta=4 \%$ & $H_{b}, \zeta=8 \%$ \\
S1 & 18 & 9 & 5 & 18 & 9 & 5 \\
S2 & 26.5 & 10 & 6.5 & 1.78 & 1.56 & 1.50 \\
S3 & 18 & 8.85 & 5.57 & 6.46 & 4.17 & 3.11 \\
S4 & 40 & 16 & 10.1 & 9.87 & 2.10 & 1.46 \\
S5 & 26.3 & 13.5 & 9.3 & 3 & 0.9 & 0.65 \\
\hline
\end{tabular}

the signals from S2 to S5, the maximum voltage increases from $3.2 \mathrm{~V}, 10 \mathrm{~V}, 5 \mathrm{~V}, 0.65 \mathrm{~V}$ to $15 \mathrm{~V}$, $12.7 \mathrm{~V}, 22 \mathrm{~V}, 29 \mathrm{~V}$, respectively. Moreover, for $\zeta=8 \%$ and the signals from S2 to S5, the RMS value of the voltage increases from $1.5 \mathrm{~V}, 3.11 \mathrm{~V}, 1.46 \mathrm{~V}, 0.65 \mathrm{~V}$ to $6.5 \mathrm{~V}, 5.57 \mathrm{~V}, 10.1 \mathrm{~V}$ and 9.3 V, respectively. Further values are provided in Table 5 and Table 6.

\subsubsection{Influence of the damping}

In Figure 14, the optimized thickness $H_{o}$ for each signal (from S1 to S5) is used and damping values equal to $0 \%, 2 \%$ and $4 \%$ are considered. It is here observed that different values of damping can be obtained creating a coarse vacuum box that accommodates the bimorphs. Conversely, $H_{b}$ is considered in Figure 15. For damping values equal to $0 \%, 4 \%, 8 \%$ and considering the signal S2, the RMS value of the voltage increases from $6.5 \mathrm{~V}$ to $10 \mathrm{~V}$ up to $26.5 \mathrm{~V}$. The peak value of $107 \mathrm{~V}$ corresponds to signal S5 when $\zeta=0 \%$. The maximum RMS value is obtained for signal S4 and it is equal to $40 \mathrm{~V}, 16 \mathrm{~V}$ and $10.1 \mathrm{~V}$ for damping values equal to $0 \%, 4 \%, 8 \%$, respectively. It is clear that mechanical damping can remarkably affect the output voltage for vibration frequencies close to the resonance condition (see Figure 15). Conversely, the influence of the damping is relatively small for the non-resonance case, see Table 5, Table 6 and Figure 16. For signal S3, the peak voltage increases from $12.7 \mathrm{~V}(4 \%)$ to $51 \mathrm{~V}(0 \%)$ when the thickness is optimized while it changes from $10 \mathrm{~V}(\zeta=4 \%)$ to $20 \mathrm{~V}(\zeta=0 \%)$ when the thickness is $H_{b}$.

\subsubsection{A note on beating effects}

The beat phenomenon occurs when a dynamic undamped system with a proper eigenfrequency is forced with an oscillating external load having a frequency slightly different, i.e. it is excited in a condition close to the resonance. In this work, we considered a $5 \%$ mismatch between the natural frequency $f_{1}$ of the bimorph and the cable frequency $f_{c}$ with the highest amplitude in the signal spectrum. Consequently, if we focus on the results for the undamped system (black lines), two waves are visible in the time histories of, both, tip displacement and electrical voltage, travelling with frequencies approximately equal to $f_{t}=\left|\left(f_{c}+f_{1}\right) / 2\right|$ and $f_{s}=\left|\left(f_{c}-f_{1}\right) / 2\right|$, respectively. When $f_{1}$ is equal to $f_{c}$, the system oscillates in phase with the external vibration and, therefore, 
Table 7: Bimorph voltage output $[\mathrm{V}]$ for different frequency shifts $(\zeta=8 \%)$.

\begin{tabular}{llll}
\hline & Max & RMS & Shift (\%) \\
S5 & 65 & 26.41 & $5 \%$ \\
S5 & 26 & 9.85 & $10 \%$ \\
\hline
\end{tabular}

Table 8: Bimorph voltage output [V] for different electrical resistance values.

\begin{tabular}{|c|c|c|c|c|c|}
\hline \multicolumn{6}{|c|}{ Time domain analysis $(\zeta=8 \%)$} \\
\hline & $0 \mathrm{k} \Omega$ & $10 \mathrm{k} \Omega$ & $50 \mathrm{k} \Omega$ & $100 \mathrm{k} \Omega$ & \\
\hline S5 & 7.67 & 9.11 & 30.85 & 41.00 & $\operatorname{Max} / \operatorname{Min}$ \\
\hline S5 & 1.75 & 2.45 & 6.23 & 11.25 & \\
\hline \multicolumn{6}{|c|}{ Frequency domain analysis $(\zeta=8 \%)$} \\
\hline & $0 \mathrm{k} \Omega$ & $10 \mathrm{k} \Omega$ & $50 \mathrm{k} \Omega$ & $100 \mathrm{k} \Omega$ & \\
\hline S5 & 1.71 & 2.34 & 4.23 & 7.49 & Max/Min \\
\hline S5 & 0.05 & 0.06 & 0.13 & 0.27 & RMS \\
\hline
\end{tabular}

the energy absorbed from the EH will increase resulting in a monothonic growth of the amplitude being $f_{s}=0$, see Figure 17a. On the other hand, if $f_{s} \neq 0$ (as common in practice) the amplitude of the first oscillation initially increases until the device is in phase with the external acceleration, and then decreases. To clarify this behaviour, we provide the output voltage for a frequency shift $\left(f_{1}-f_{c}\right)$ equal to to $5 \%$ and $10 \%$ in Figure $17 \mathrm{~b}$. For signal S5, this behaviour corresponds to a peak decrease from $65 \mathrm{~V}$ to $26.41 \mathrm{~V}$ for a mismatch equal to $5 \%$ and $10 \%$, while the RMS value decreases from $26 \mathrm{~V}$ to $9.85 \mathrm{~V}$, see Table 7 . Hence, this phenomenon can be exploited for applications at the nanoscale where the experimental evidence shows that the damping is very small.

\subsubsection{Influence of the input acceleration}

In Figure 16, dynamic voltage responses are compared for different acceleration records from S1 to S5. Additionally, the influence of mechanical damping on the dynamic voltage responses can be again inferred from Figure $16 \mathrm{~b}$ and $16 \mathrm{~d}(\zeta=4 \%)$ and in Figure $16 \mathrm{a}$ and $16 \mathrm{c}(\zeta=0 \%)$. It is here highlighted that, for $H=H_{b}$ and $\zeta=4 \%$, the maximum voltage is obtained for the signal S1 (10 $\mathrm{V})$ and not $\mathrm{S} 5(0.65 \mathrm{~V})$, even if the RMS value for the acceleration increases. This behavior is also confirmed for other damping values.

\subsubsection{Influence of the electrical resistance}

Here, the response is analysed in the frequency and time domains, considering different values for the resistor between the top bimorph layer and the ground. The analyses are limited to the most critical input acceleration S5. Three different resistance loads, namely $10 \mathrm{k} \Omega, 50 \mathrm{k} \Omega$ and $100 \mathrm{k} \Omega$ are assumed. The bimorph has a thickness $H_{f}=0.76 \mathrm{~mm}$ and a first frequency equal to 9.0747 Hz. See Figure 18 and Table 8.

\section{Conclusions}

This paper has presented a novel computational paradigm for modelling and PI of electromechanical devices employed for energy harvesting and sensing applications. The computational strategy is 
based on sensitivity feedback analysis. First, we have shown that the identification problem can be formulated by a proper set of ordinary differential equations whose equilibrium points correspond to the problem solutions. We rigorously demonstrated that this artificial dynamic system can be explicitly designed to be stable with asymptotic convergence. Moreover, the resulting equations are computationally simple enough to be easily implemented in online adaptive identification/control applications. Second, in the context of multimodal energy harvesting, the PI algorithm is used to assess the lumped coefficients of a reduced order model for an array of bimorphs implemented in real structural health monitoring conditions. We considered an array configuration with the aim to produce higher energy from broadband ambient vibrations of a cable-stayed bridge. The acceleration time-histories at different bridge locations are experimentally measured while the time histories of tip displacement/velocity and the electrical output are numerically assessed using FE simulations. The FE solutions are then used as an alternative to the experimental data to identify the lumped coefficients of the state-space model of the piezoelectric device. Finally, a numerical study is developed to characterize the response under different technological constraints and beating effects in the dynamic behaviour are also discussed. Comparative evaluations demonstrate the effectiveness of the proposed computational strategy.

\section{Acknowledgments}

Claudio Maruccio acknowledges the support from the Italian MIUR through the project FIRB Futuro in Ricerca 2010 Structural mechanics models for renewable energy applications (RBFR107AKG).
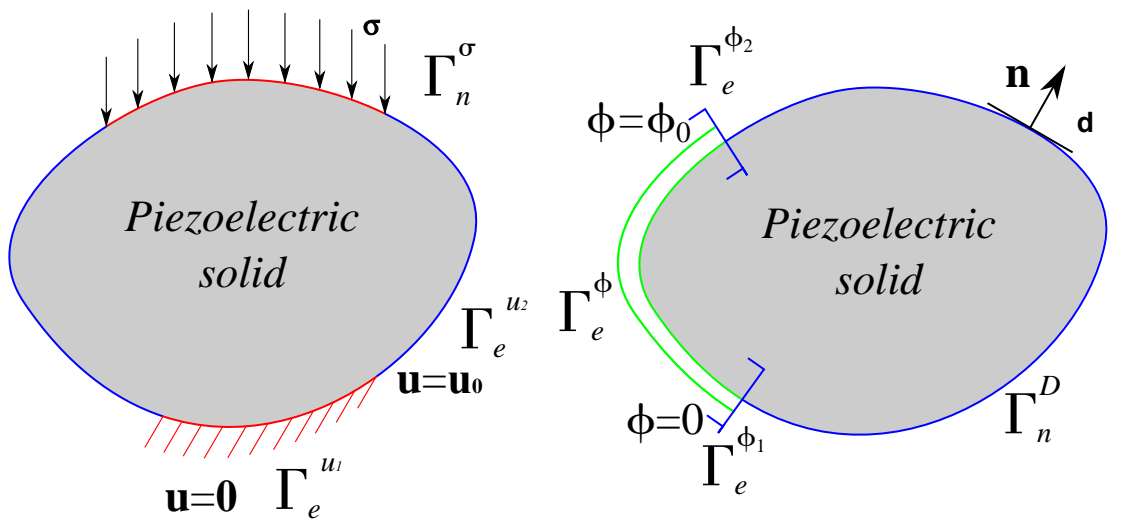

Figure 1: Boundary conditions of a piezoelectric solid. 


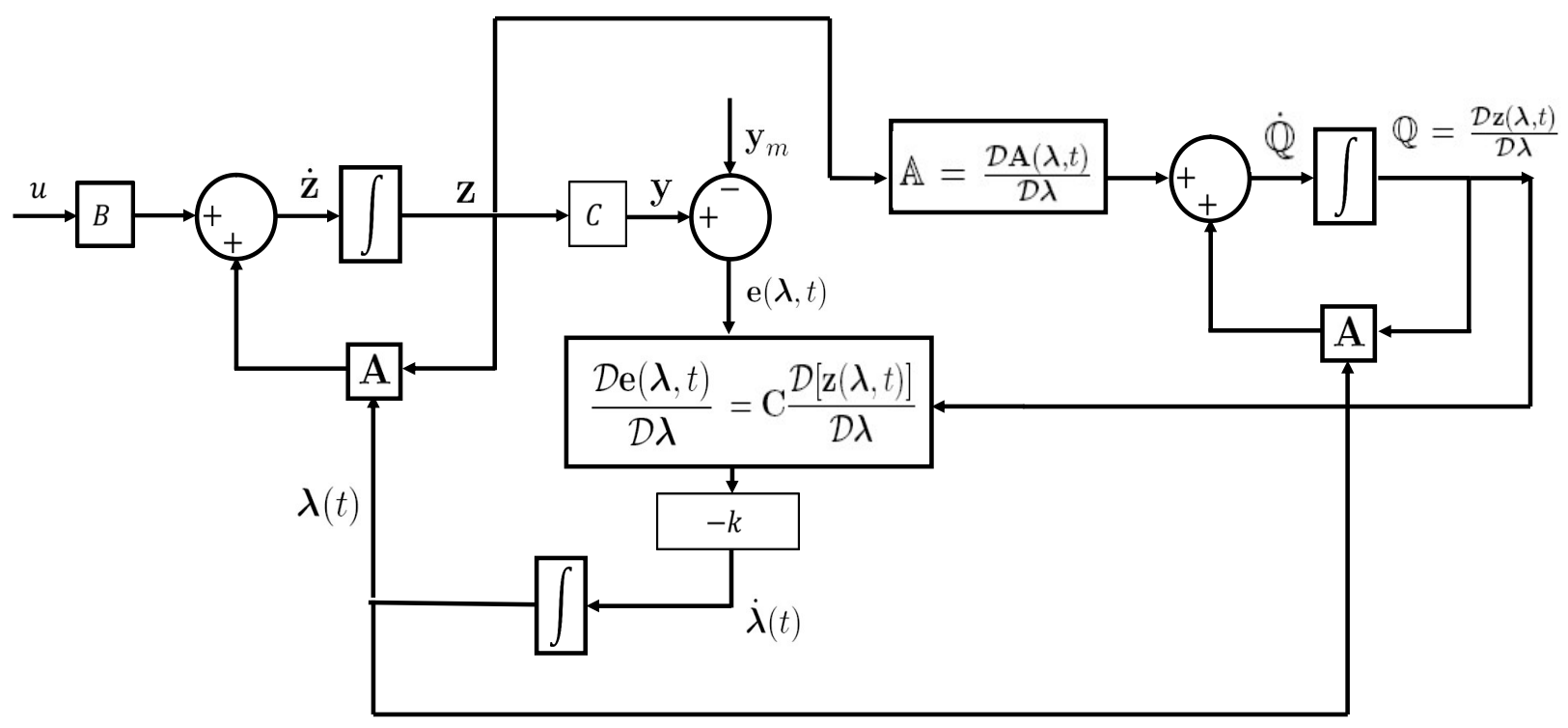

Figure 2: Block diagram.

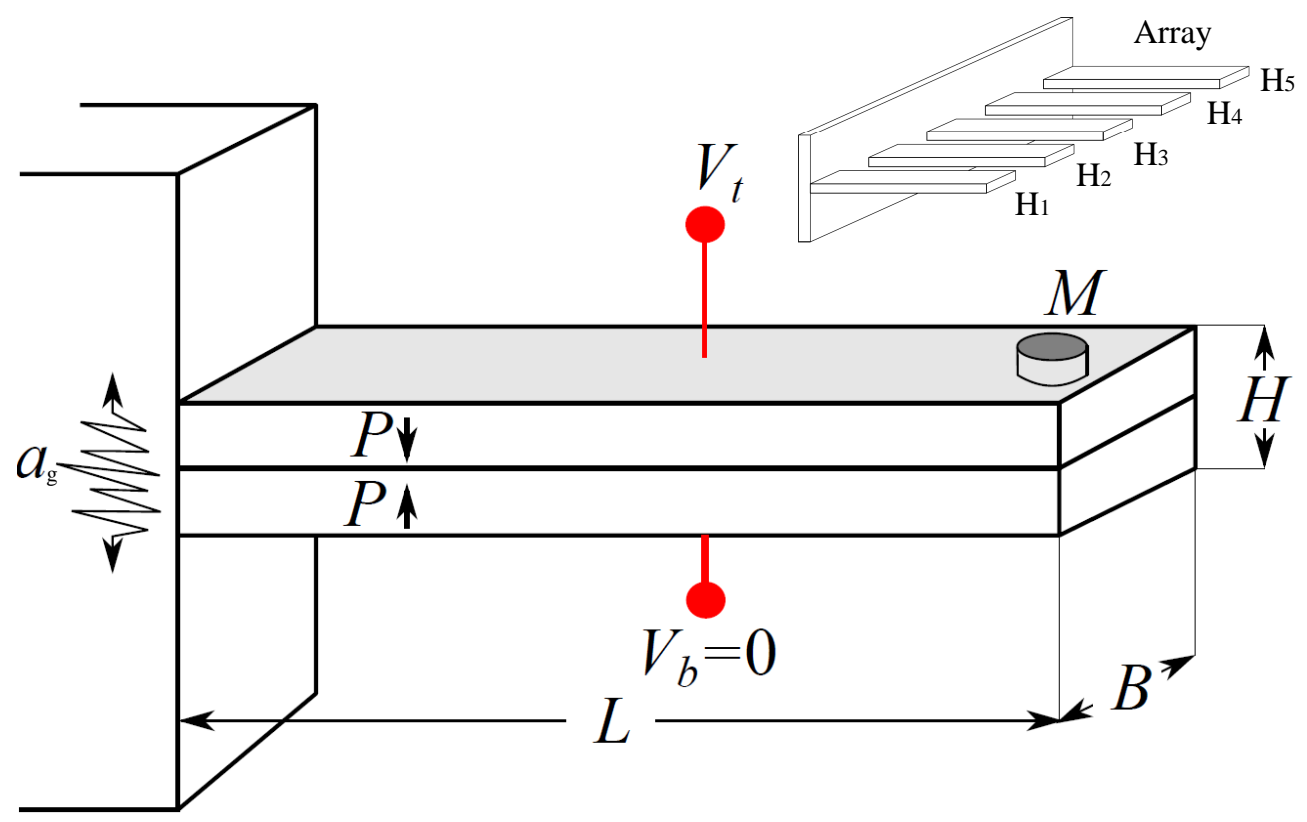

Figure 3: Piezoelectric bimorph configuration. 


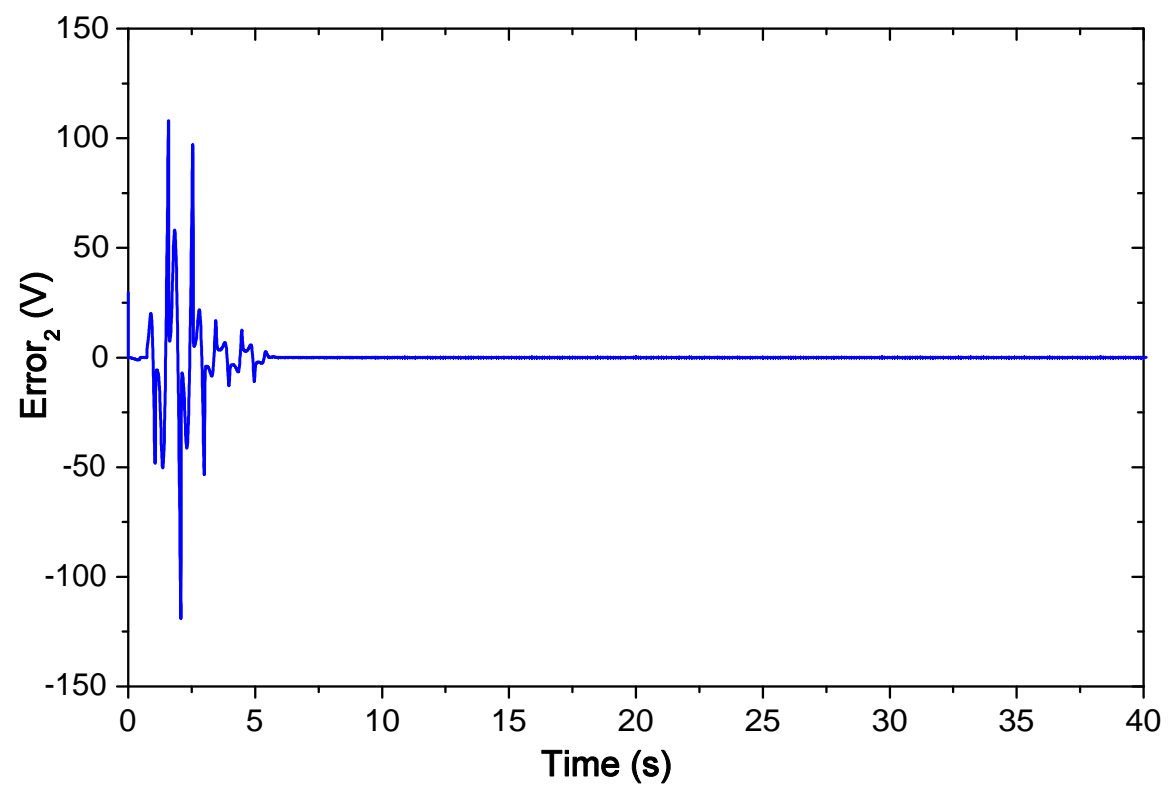

Figure 5: Relative error $v(\boldsymbol{\lambda}, t)-y_{m, 2}(t)$.

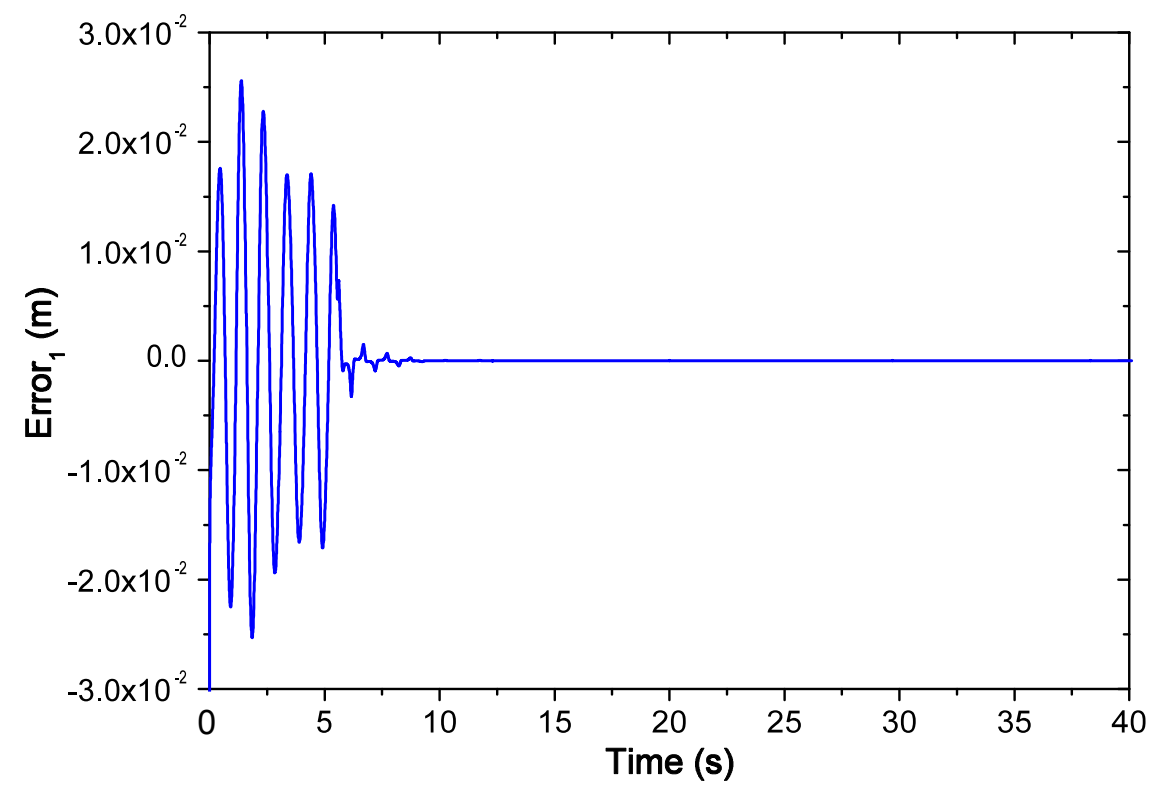

Figure 4: Relative error $\eta_{1}(\boldsymbol{\lambda}, t)-y_{m, 1}(t)$. 


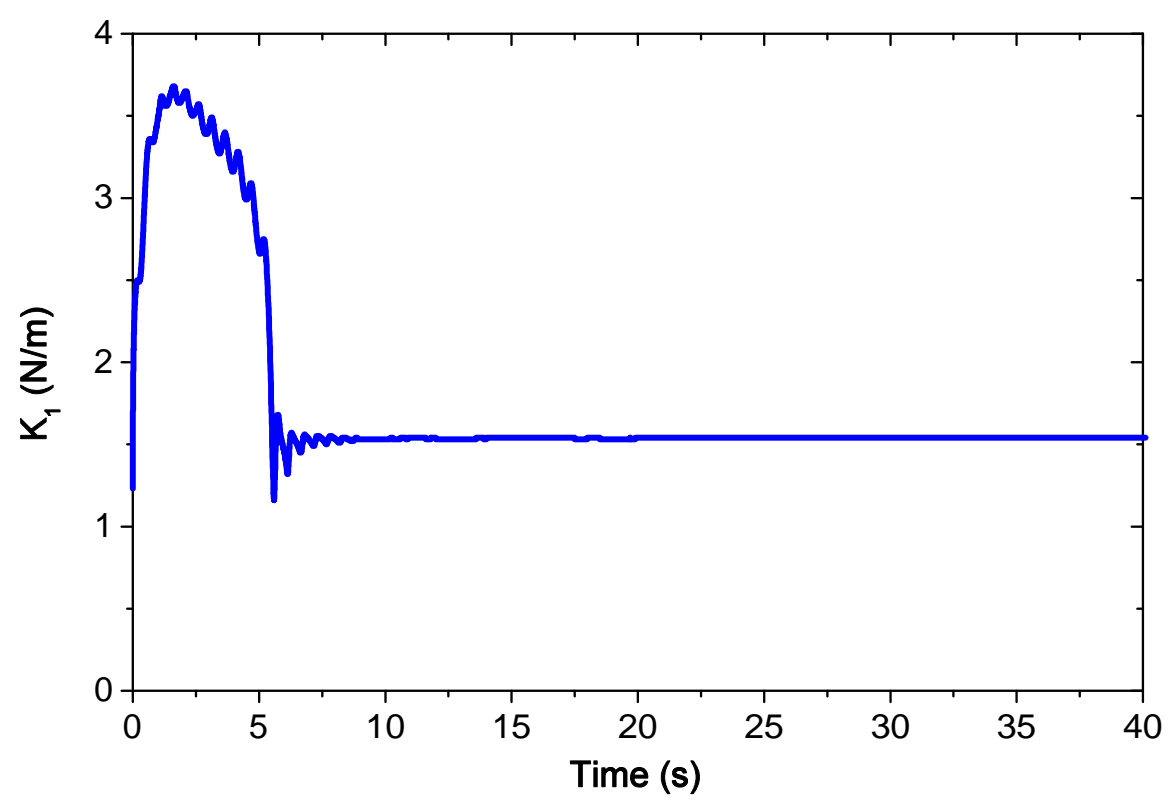

Figure 6: Convergence of $K_{1}$.

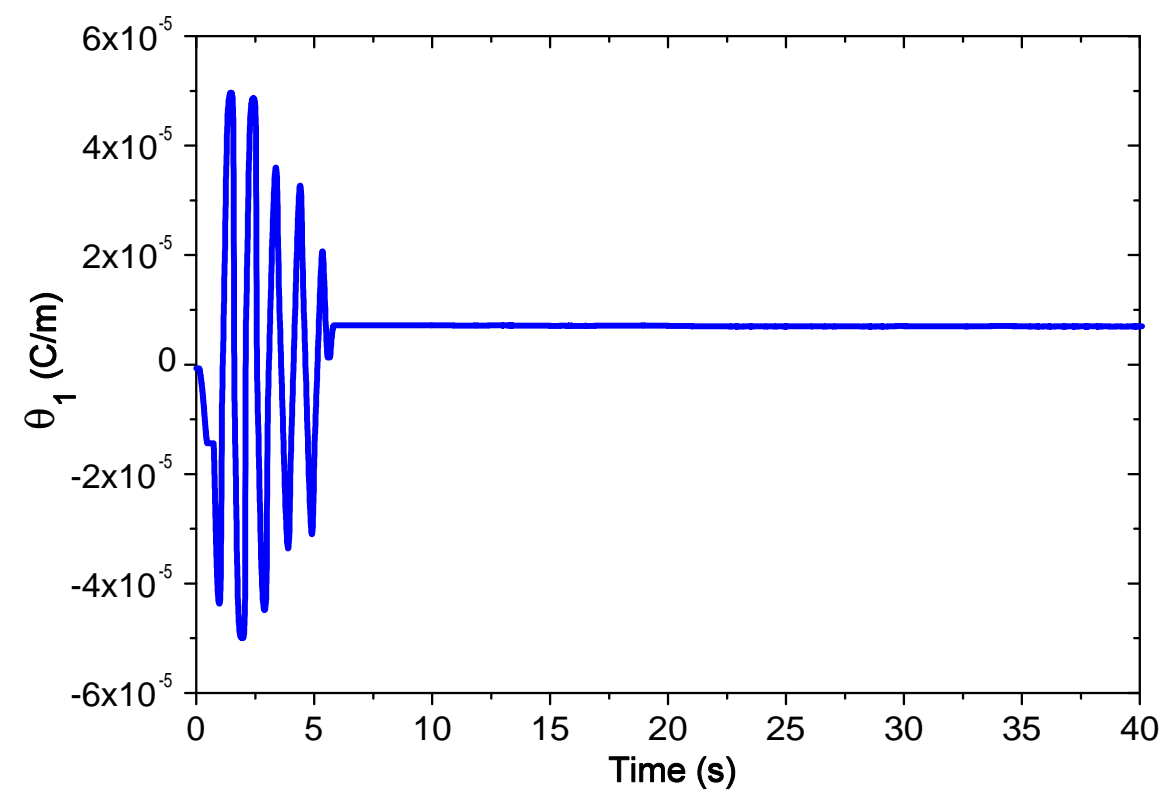

Figure 7: Convergence of $\theta_{1}$. 

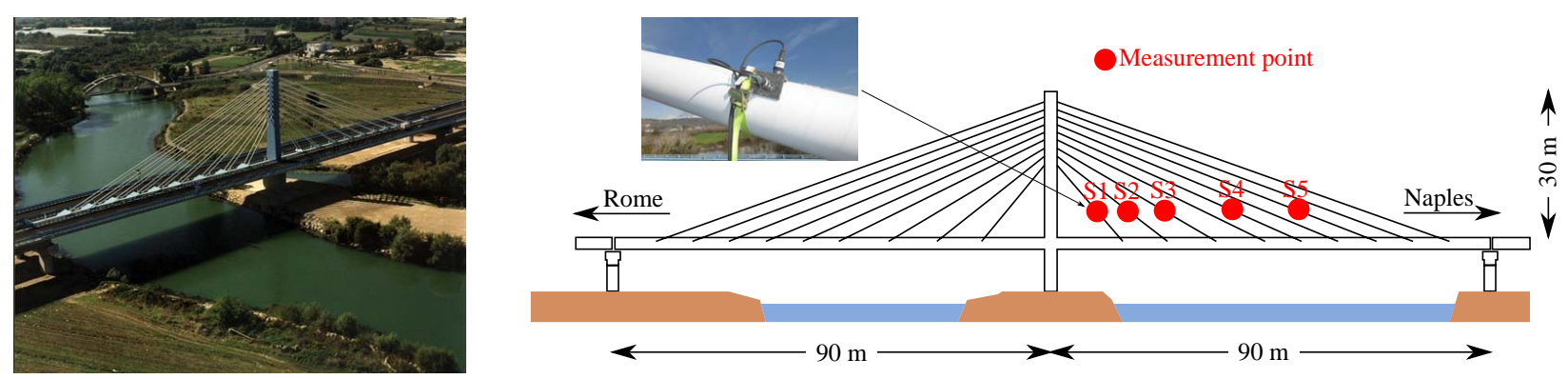

Figure 8: A photograph of the cable-stayed bridge over the Garigliano river (left), main geometrical features of the structure, selected measurement points and a photograph of the accelerometers (right).

\section{References}

[1] Hoang, N. S., Ramm, A. G. (2010). Dynamical systems method for solving nonlinear equations with monotone operators. American Mathematical Society, Mathematics of Computation, Vol.79(269), pp. 239-249.

[2] Ramm A. G., Hoang S. N. (2011). Dynamical Systems Method and Applications: Theoretical Developments and Numerical Examples, Wiley.

[3] Alexander G. Ramm (2007). Dynamical Systems Method for Solving Operator Equations, Elsevier.

[4] Zadeh, L. (1956). On the Identification Problem. IRE Transactions on Circuit Theory, Vol.3(4), pp. 277-281.

[5] Keshavarz, M., Mojra, A. (2015). Dynamic modeling of breast tissue with application of model reference adaptive system identification technique based on clinical robot-assisted palpation. Journal of the Mechanical Behavior of Biomedical Materials.

[6] Kan Liu, Qiao Zhang, Zi-Qiang Zhu, Jing Zhang, An-Wen Shen, Paul Stewart (2010). Comparison of two novel MRAS based strategies for identifying parameters in permanent magnet synchronous motors. International Journal of Automation and Computing, Vol.7(4), pp. 516524.

[7] Gatto, G., Marongiu I., Serpi A. (2013). Discrete Time Parameter Identification of a Surface Mounted Permanent Magnet Synchronous Machine. IEEE Transactions on Industrial Electronics, Vol.60(11), pp. 4869-4880.

[8] Shi Yang and Fang Huazhen (2010). Kalman filter-based identification for systems with randomly missing measurements in a network environment. Taylor and Francis Group, International Journal of Control, Vol.83(3), pp. 538-551.

[9] Ding, F., Liu, Y. and Bao, B. (2012). Gradient based and least squares based iterative estimation algorithms for multi input multi output systems. Proc. of the Institution of Mechanical Engineers Part I: Journal of Systems and Control Engineering, Vol.226(1), pp. 43-55.

[10] Dongqing Wang and Feng Ding (2011). Least squares based and gradient based iterative identification for Wiener nonlinear systems. Elsevier Science, Signal Processing, Vol.91(5), pp. 11821189. 


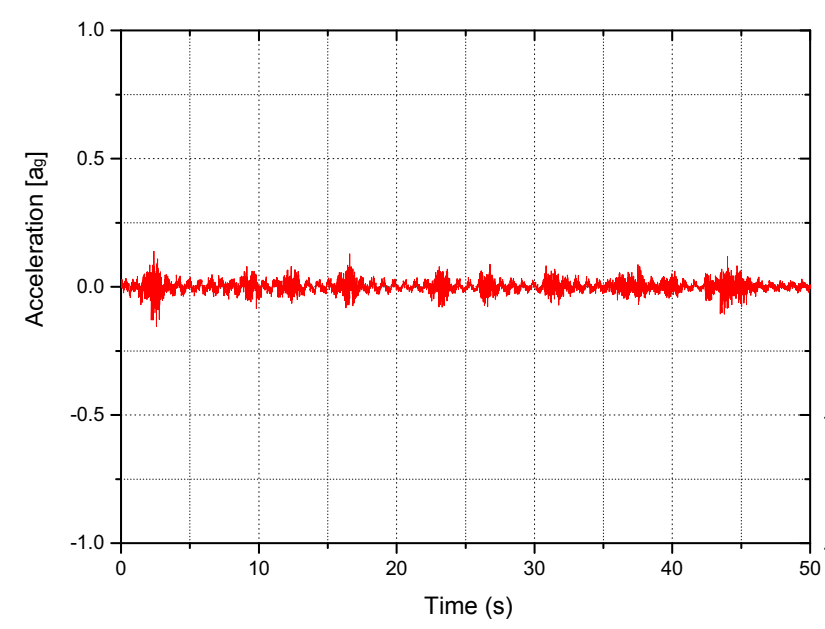

(a) $\mathrm{S} 1$

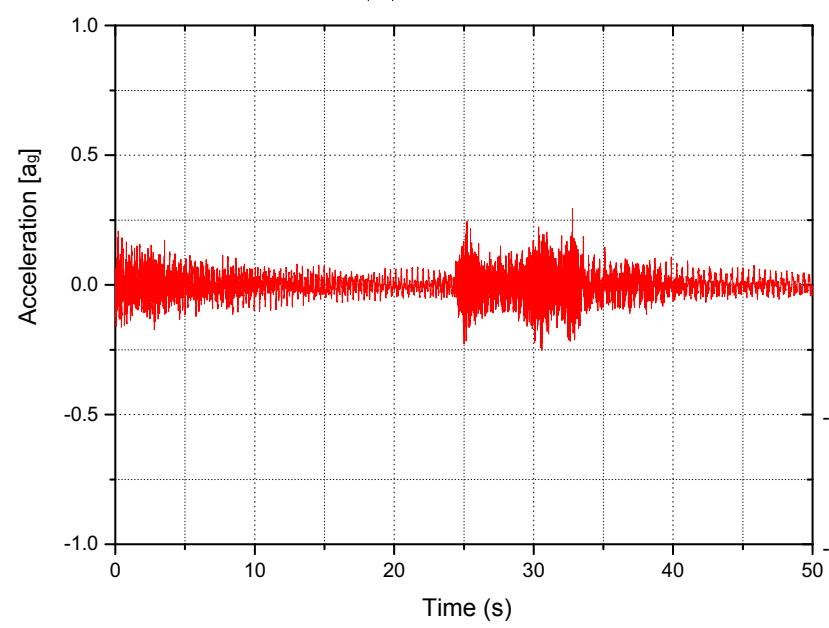

(c) S3

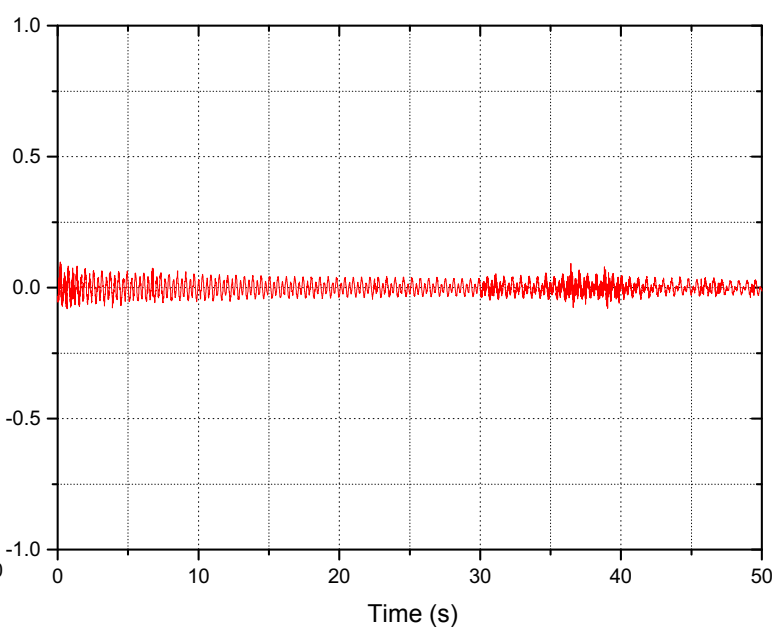

(b) $\mathrm{S} 2$

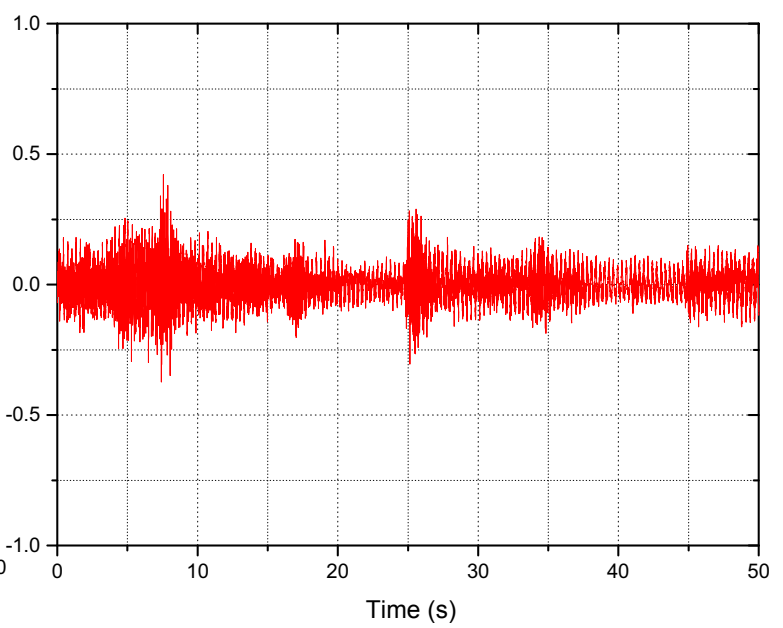

(d) S4

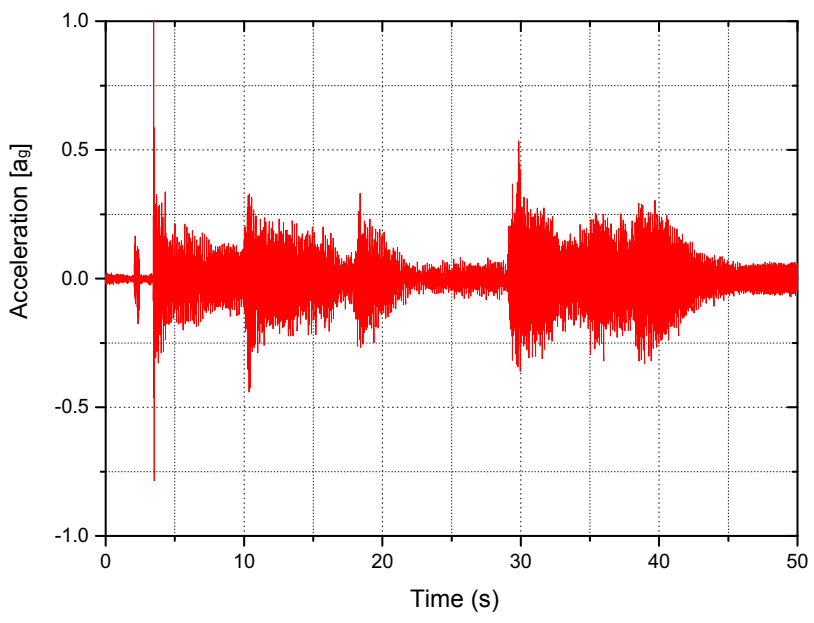

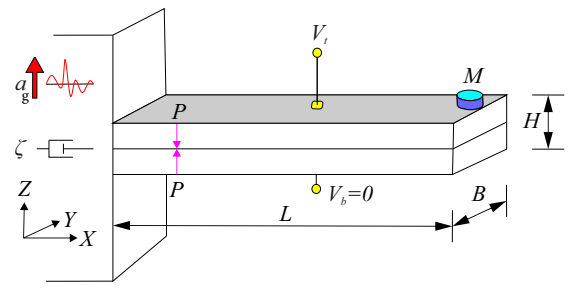

(f) Bimorph

(e) S5

Figure 9: Acceleration data (time domain). 


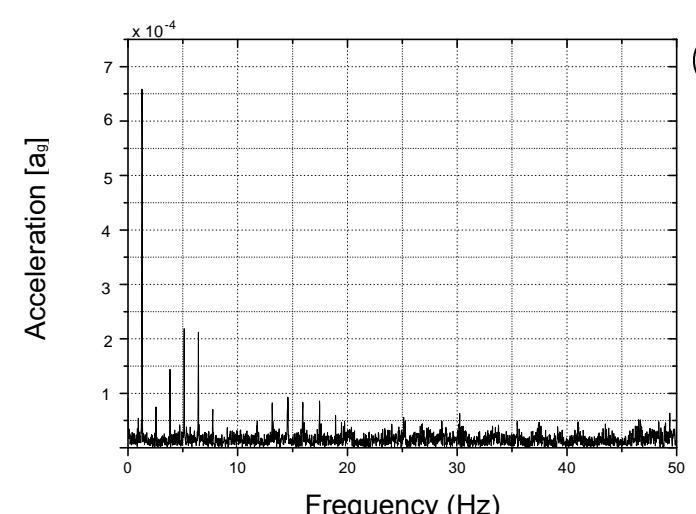

(a) $\mathrm{S} 1$

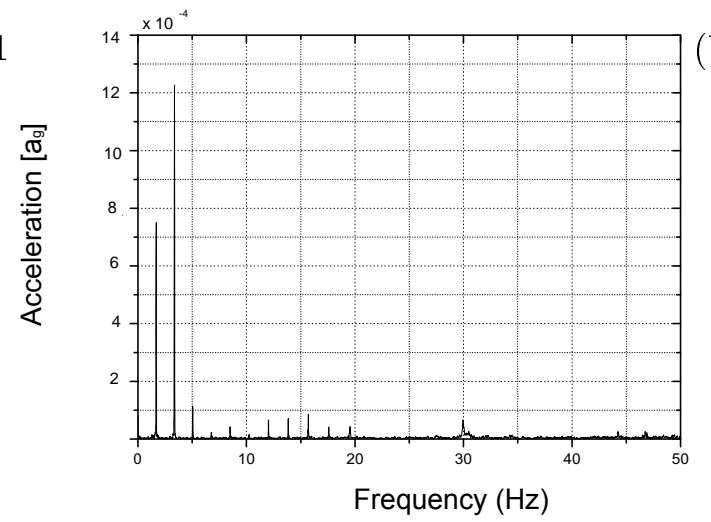

(b) S2

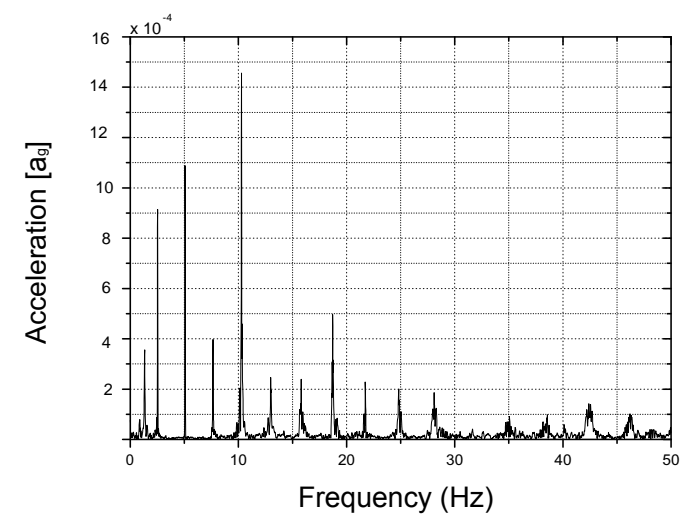

(c) S3
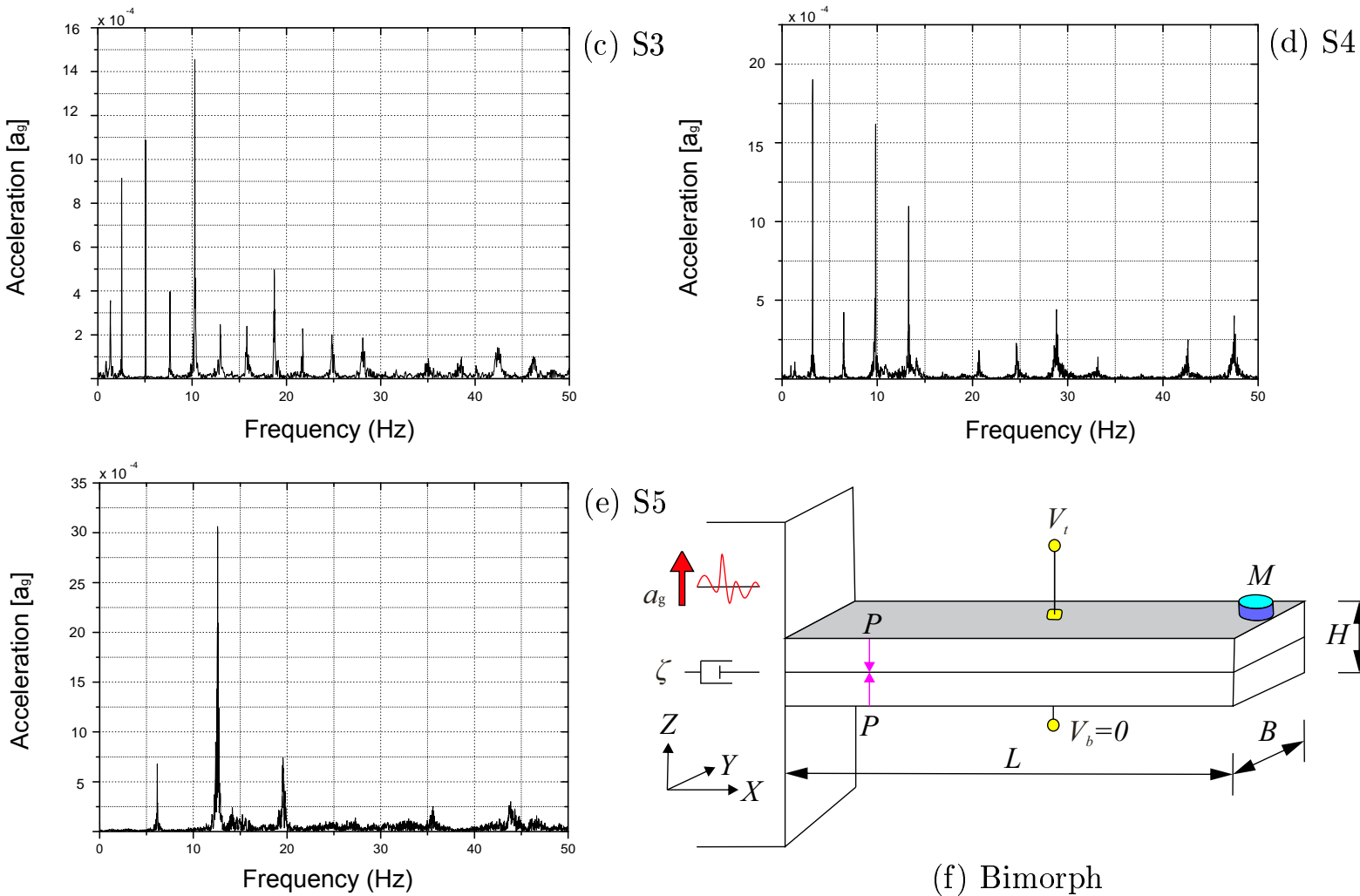

(e) $\mathrm{S} 5$

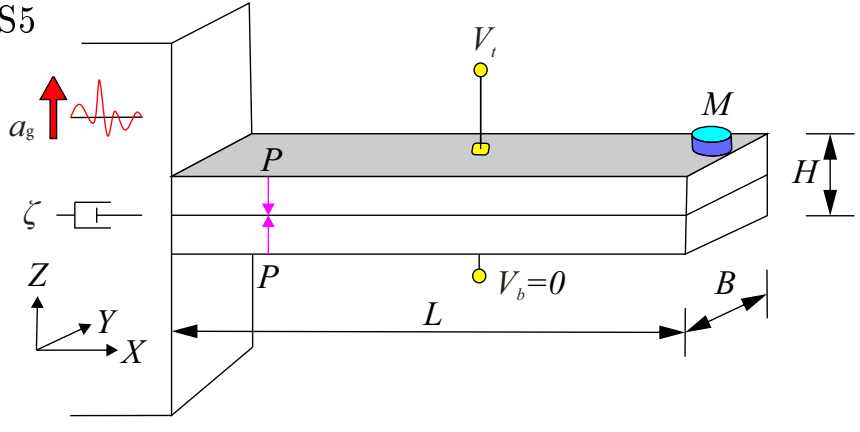

(f) Bimorph

Figure 10: Acceleration data (frequency domain). 
(a) $\mathrm{S} 2$
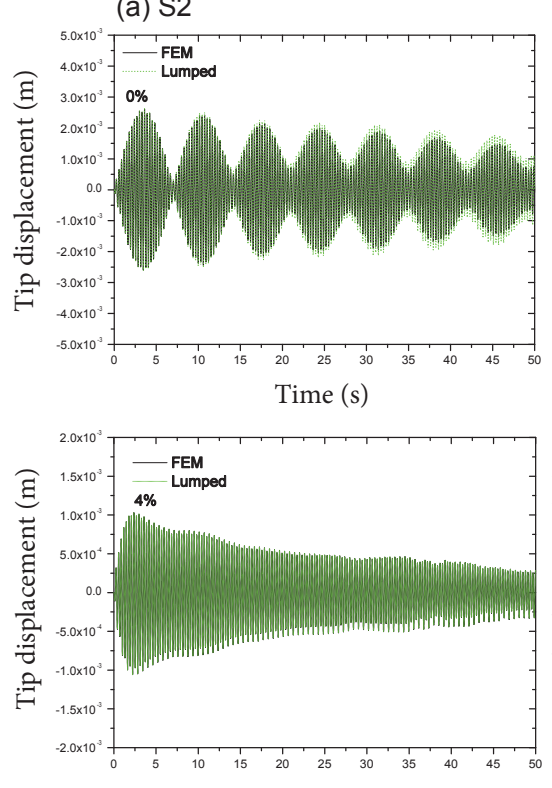

Time (s)

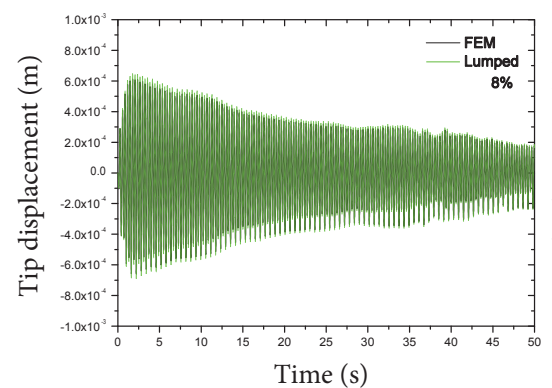

Figure 11: Comparison of finite element model and lumped parameters model (tip displacement).
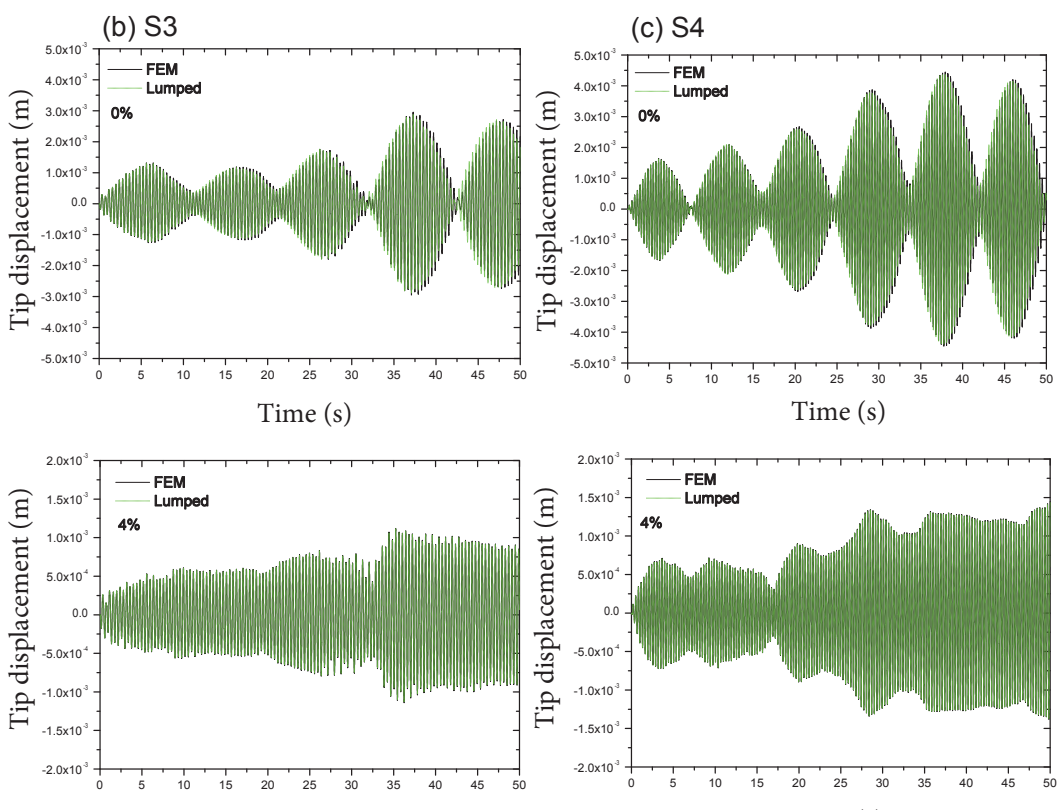

Time (s)
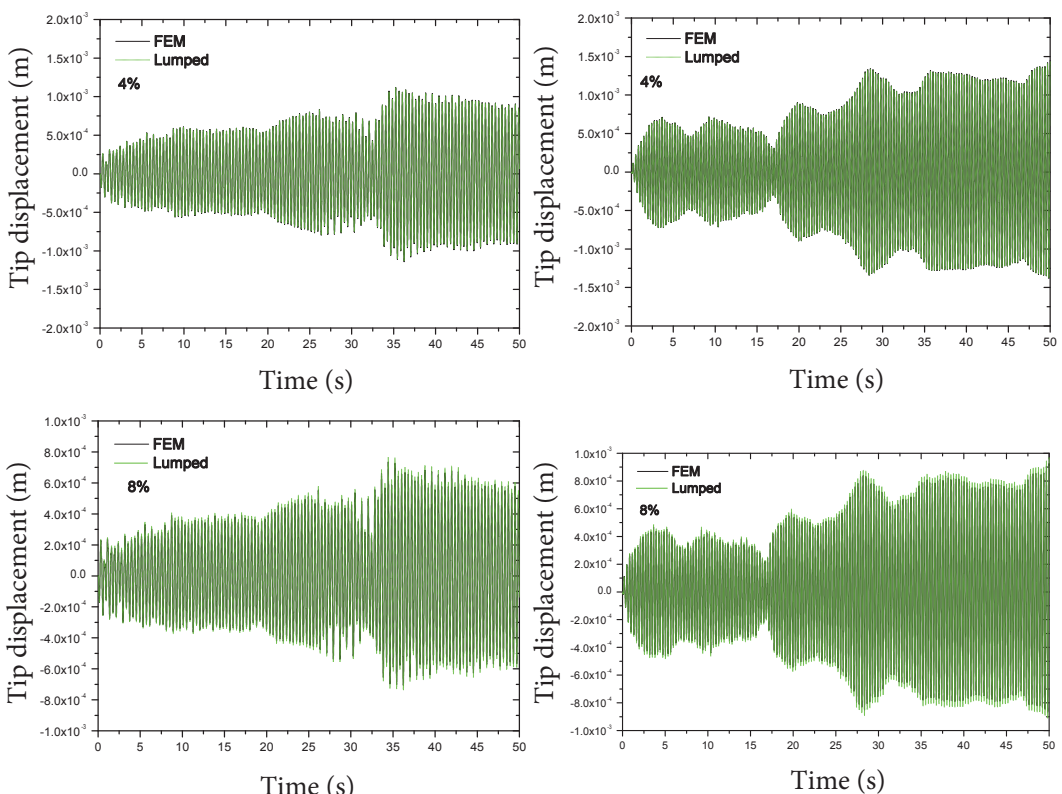
(a) $\mathrm{S} 2$
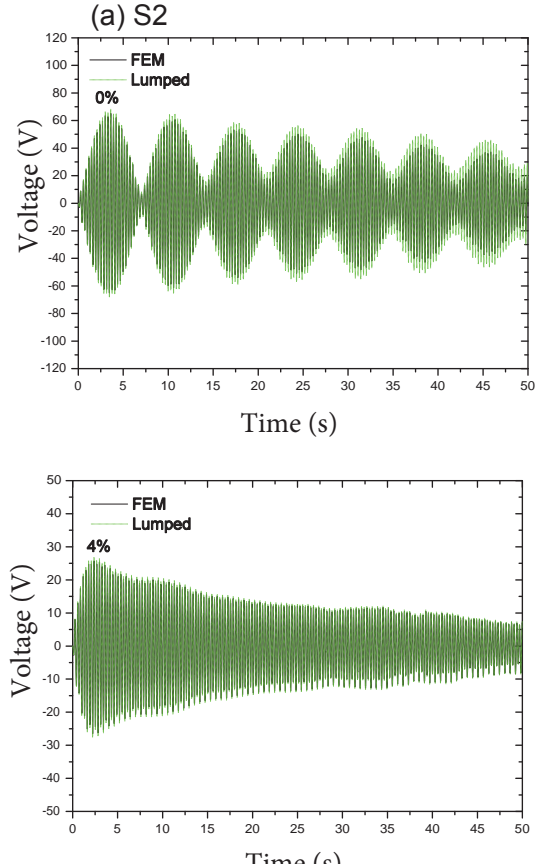

Time (s)

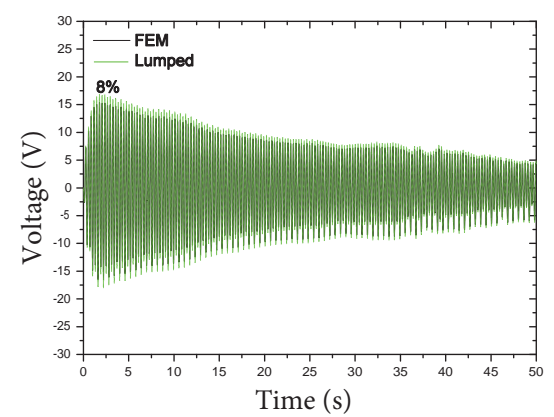

(b) S3
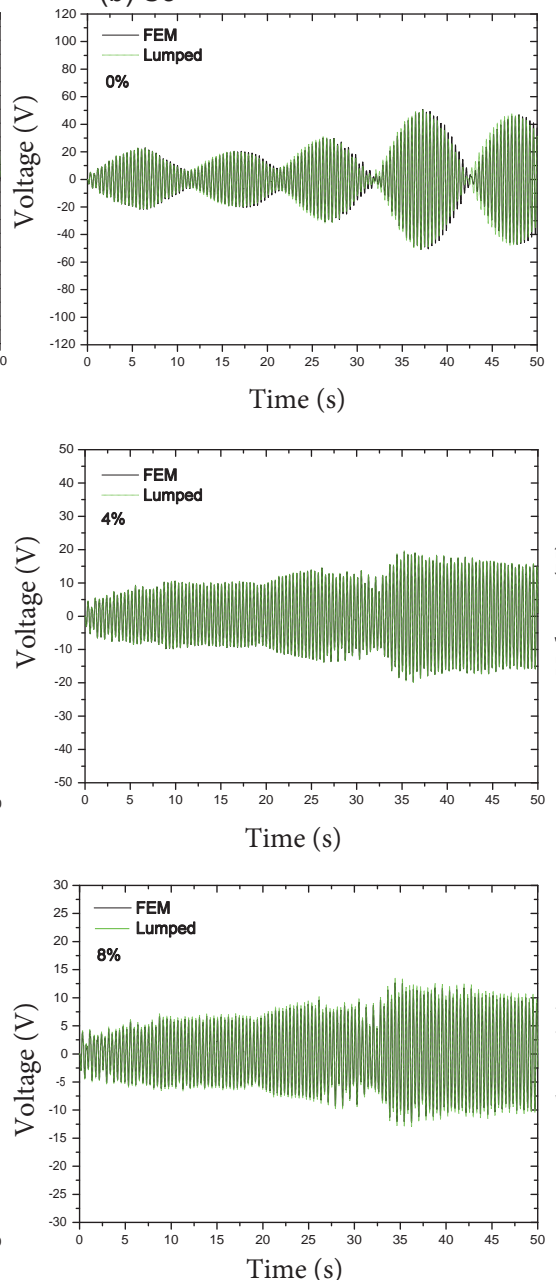

(c) S4
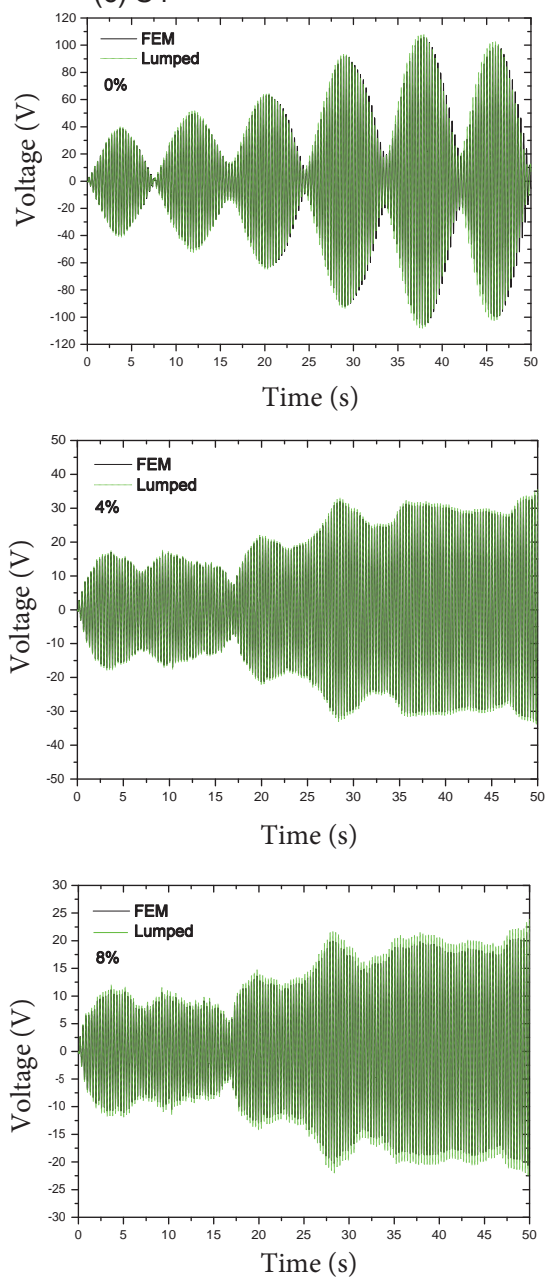

Figure 12: Comparison of finite element model and lumped parameters model (voltage on the electrodes). 
(a) $\mathrm{S} 1$

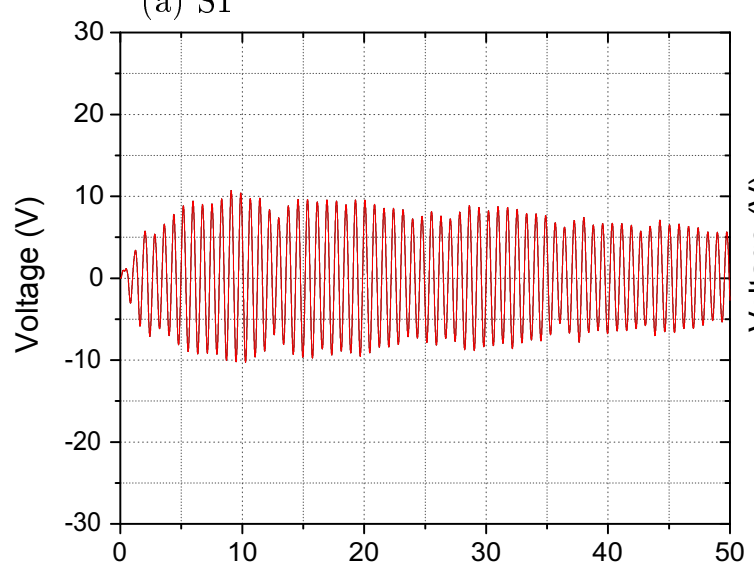

(c) $\mathrm{S} 3$

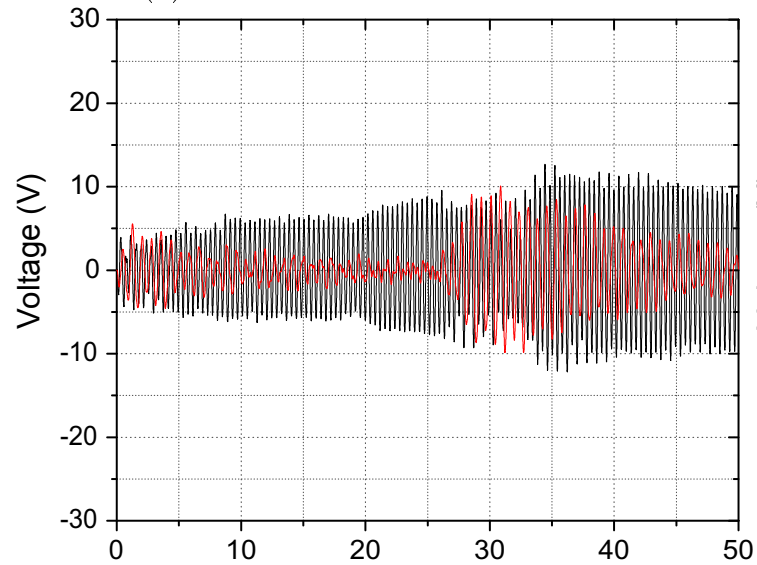

(e) S5

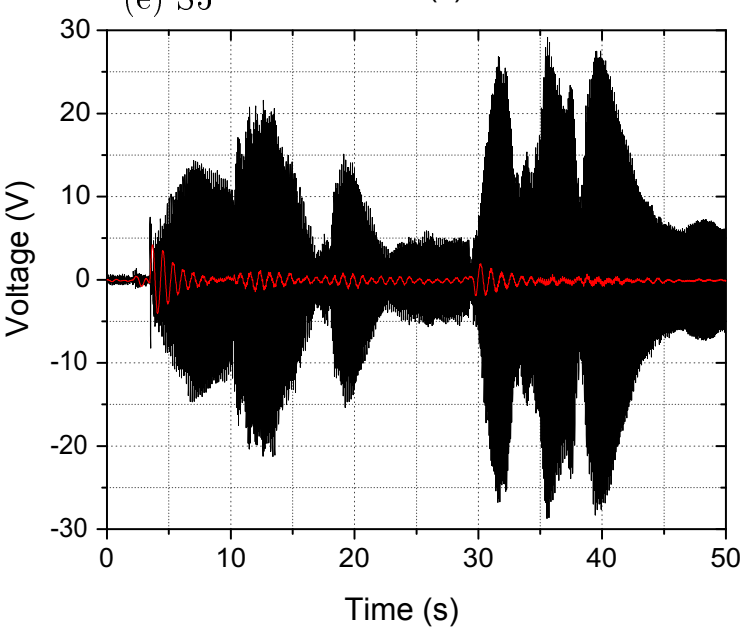

(b) S2

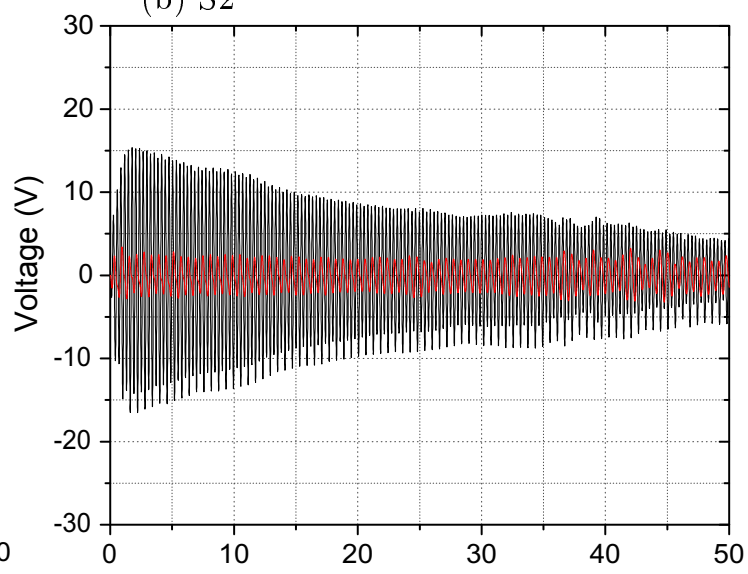

(d) $\mathrm{S} 4$

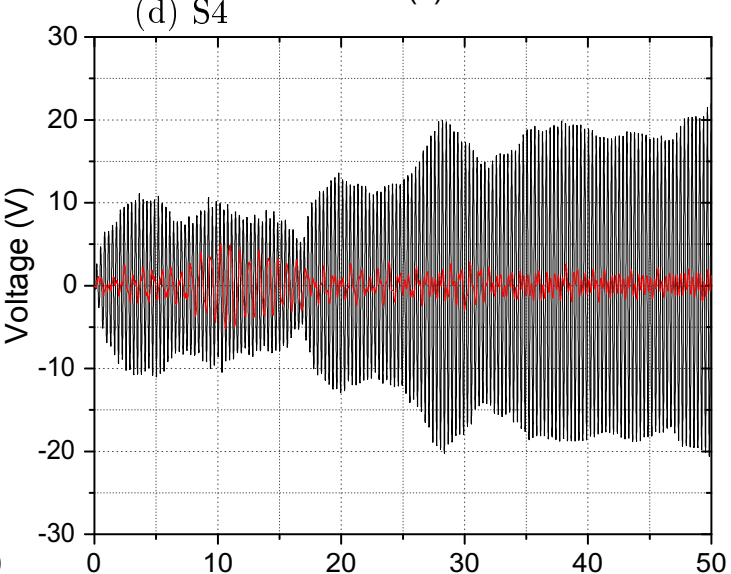

Time (s)

(f) Bimorph

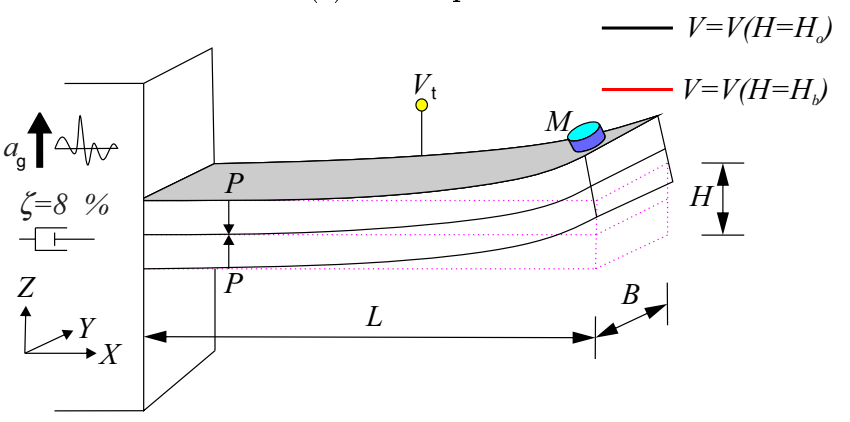

Figure 13: Sensitivity to geometry. 
(a) S1

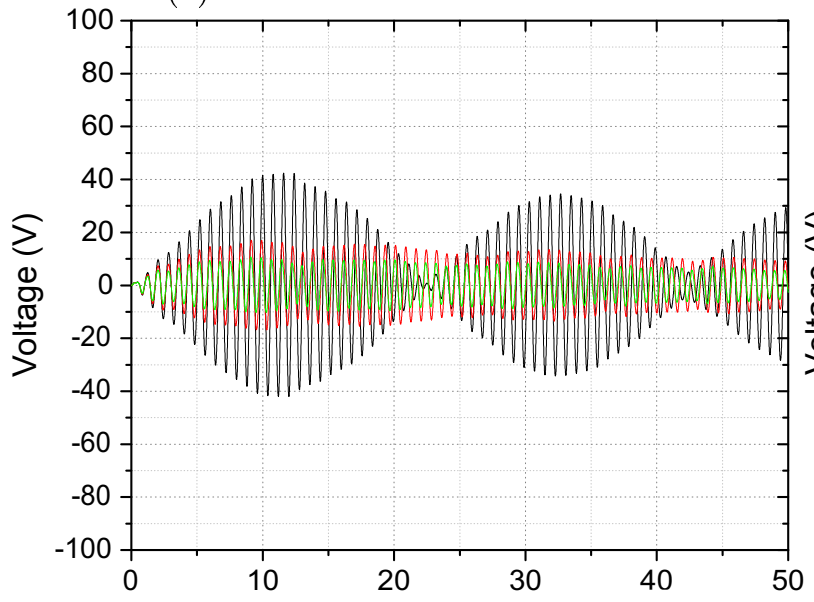

(c) S3

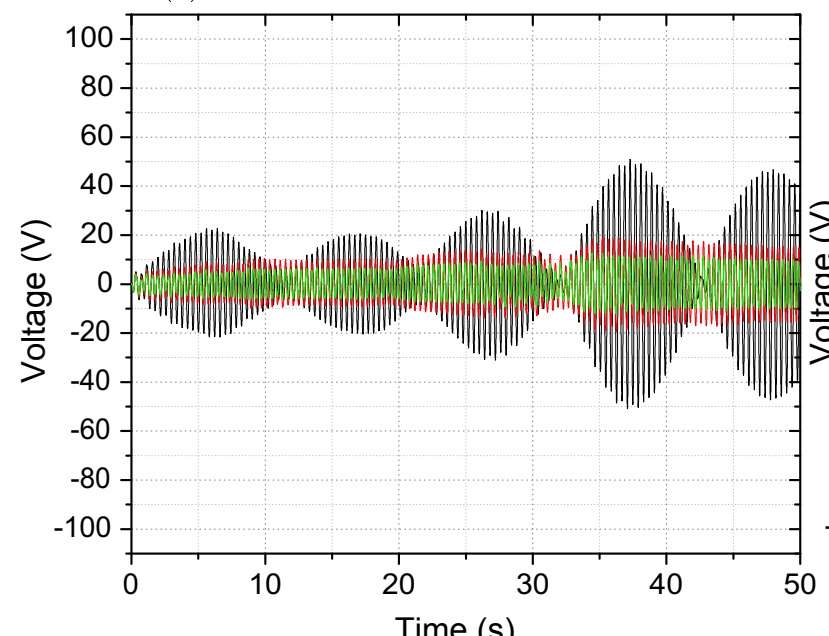

(e) $\mathrm{S} 5$

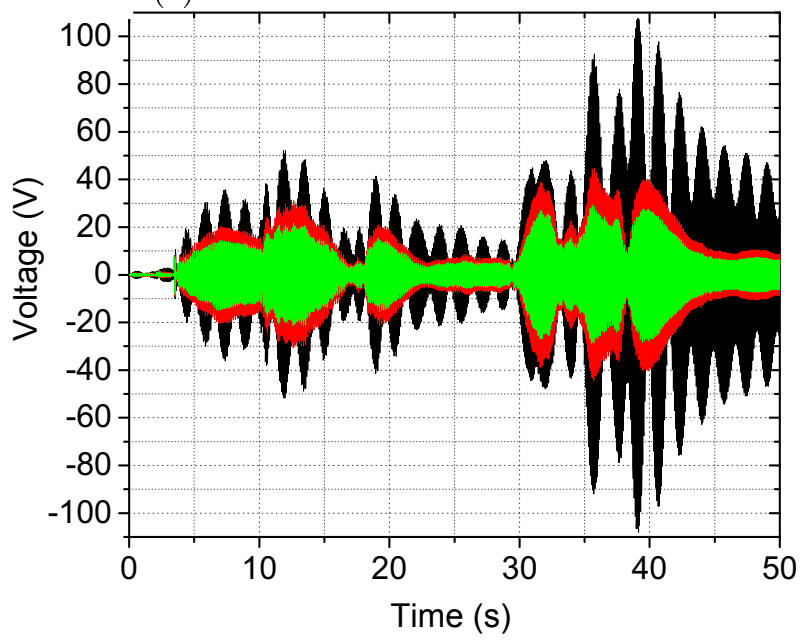

(b) S2

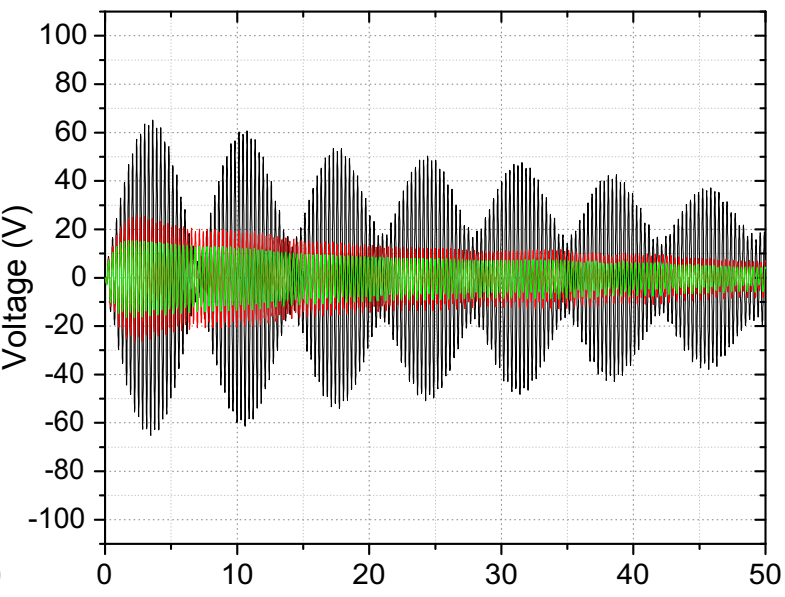

(d) $\mathrm{S} 4$

Time (s)

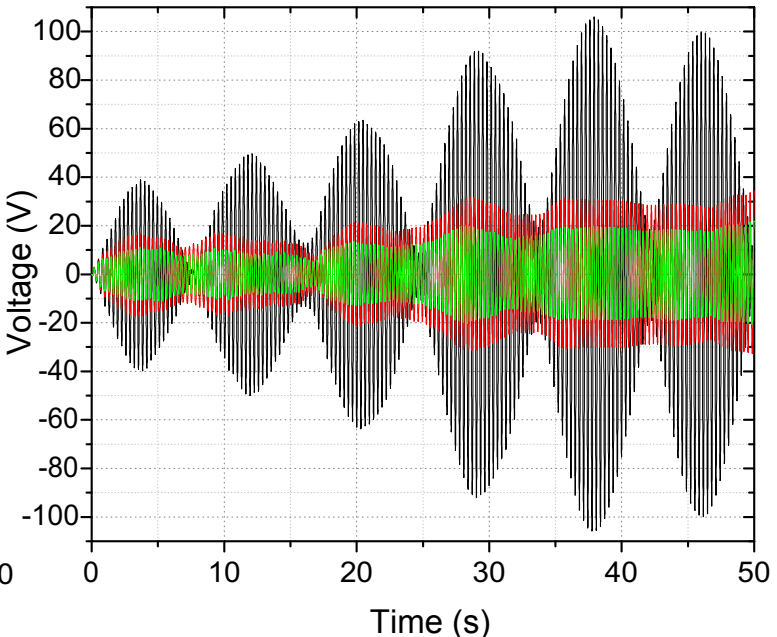

(f) Bimorph $\quad-V=V(\zeta=0 \%)$

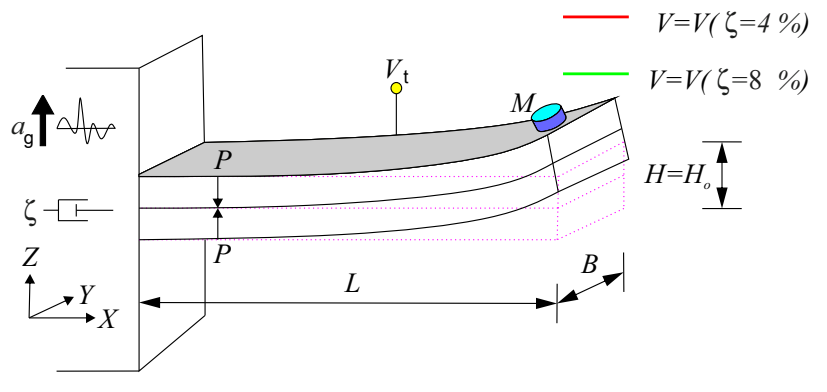

Figure 14: Sensitivity to damping $\left(H_{o}\right)$. 
(a) S1

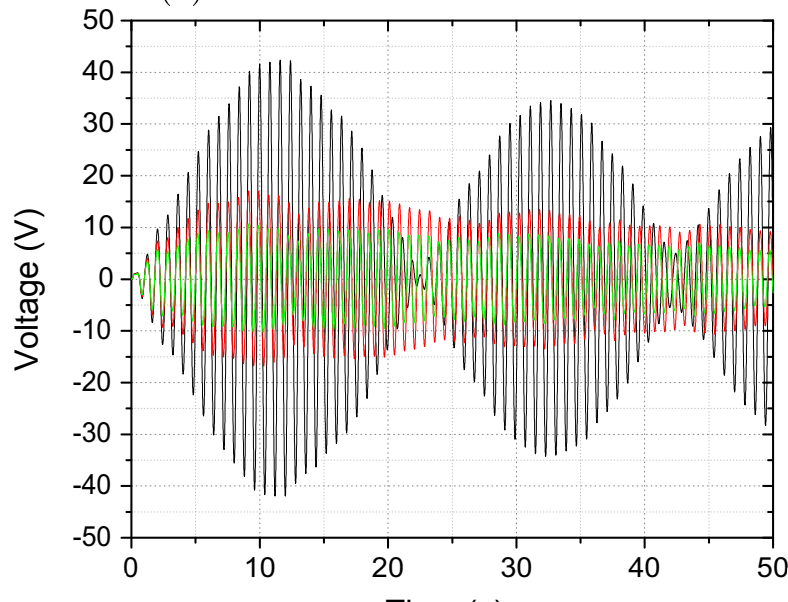

(c) $\mathrm{S} 3$

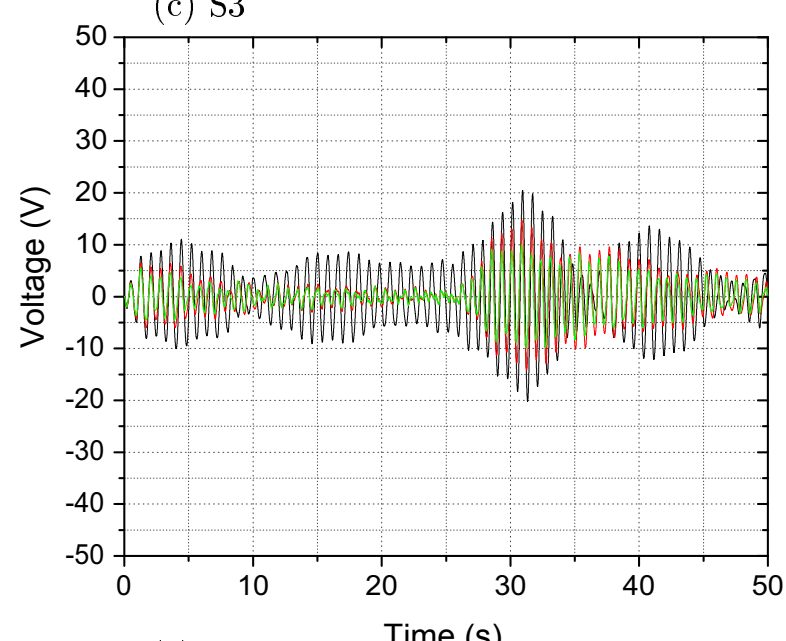

(e) S5

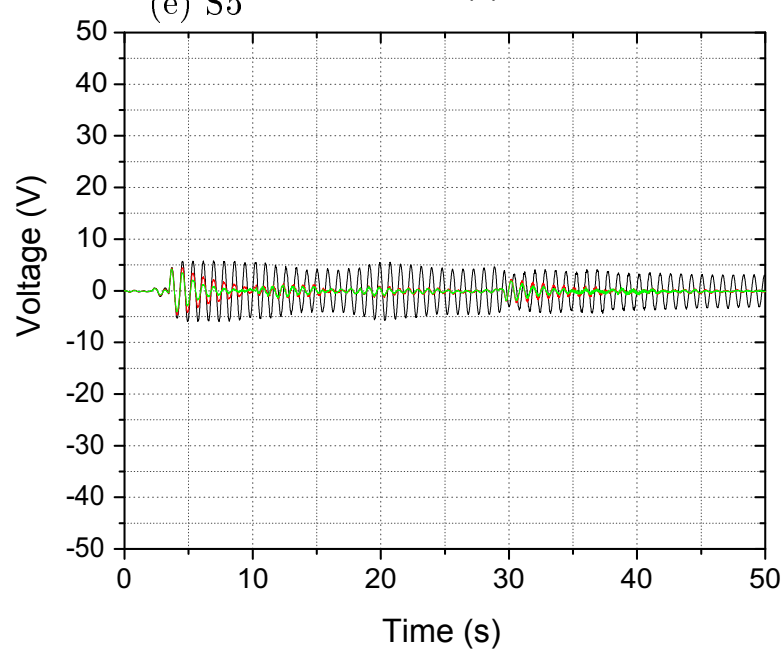

(b) S2

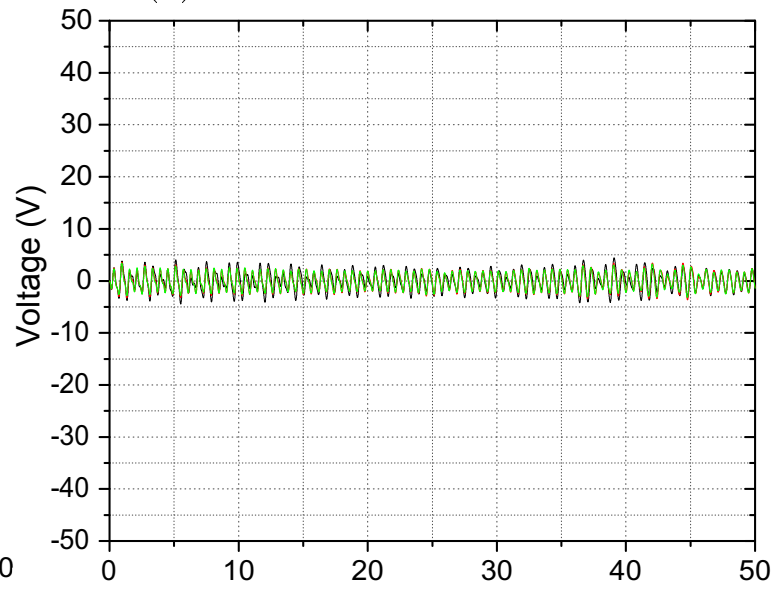

(d) S4 Time (s)

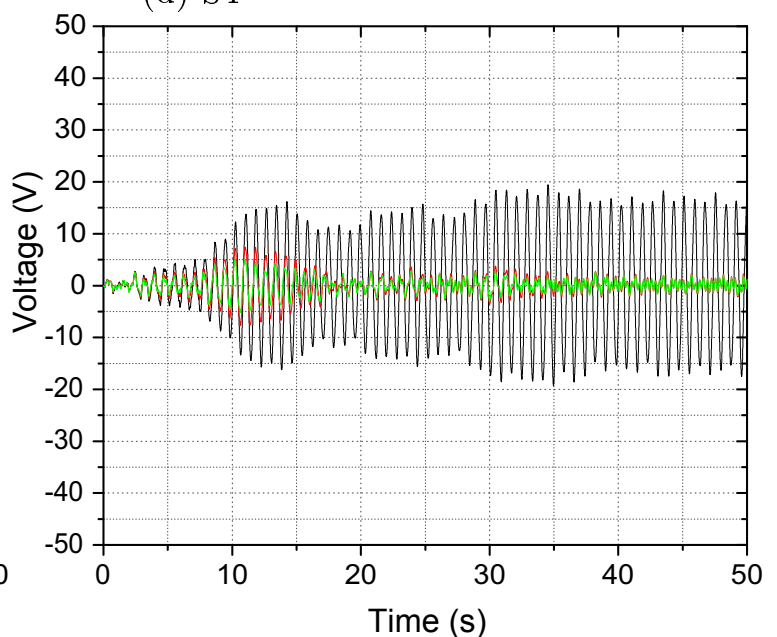

Time (s) $\longrightarrow V=V(\zeta=0 \%)$

(f) Bimorph

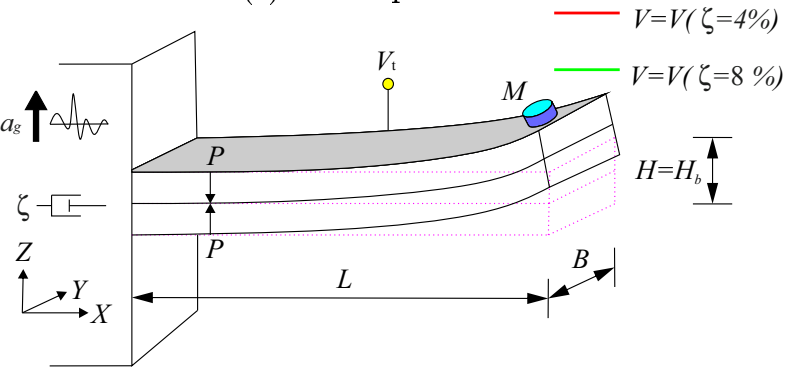

Figure 15: Sensitivity to damping $\left(H_{b}\right)$. 

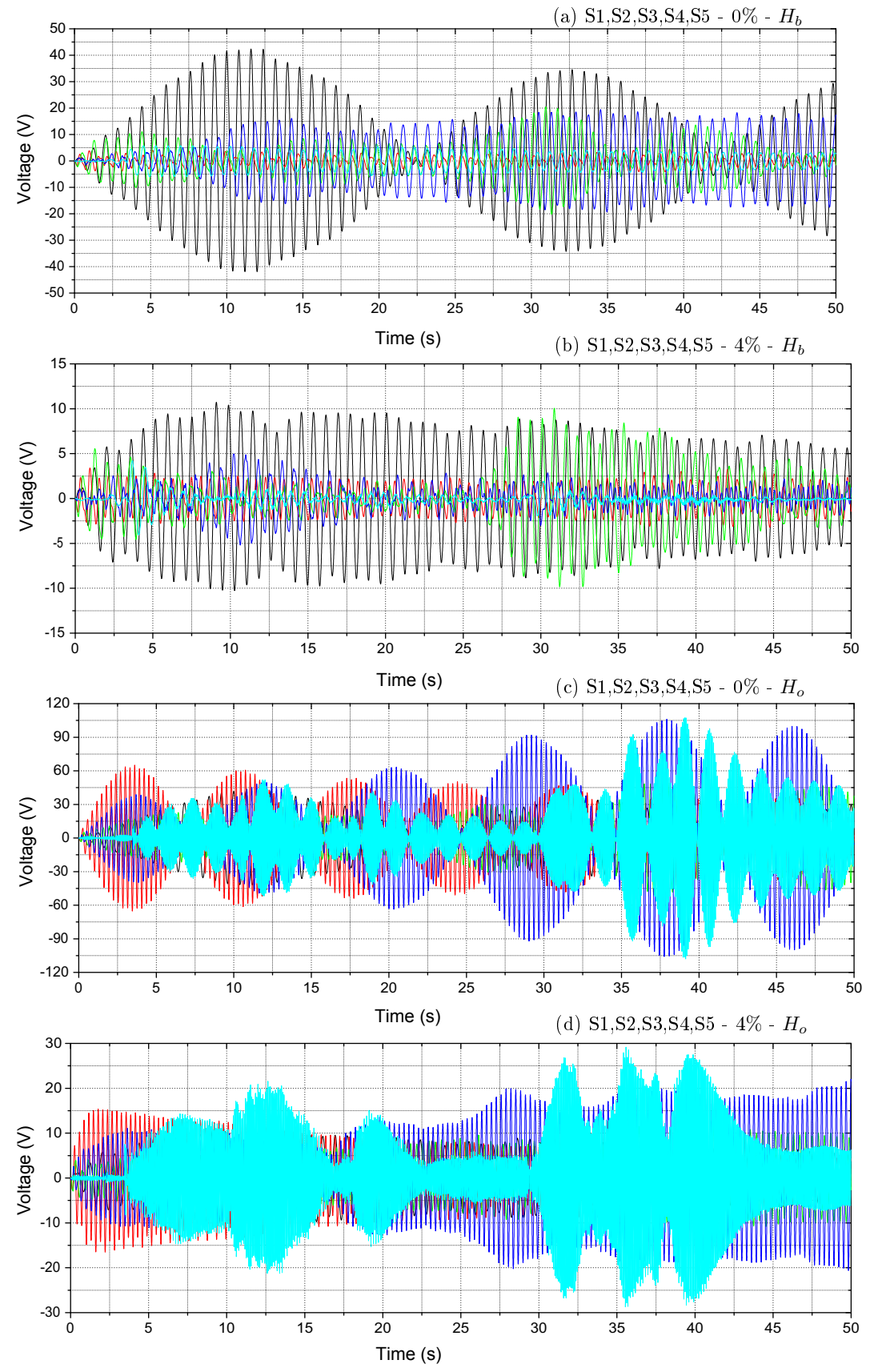

Figure 16: Sensitivity to input acceleration. 
(a) Resonant case

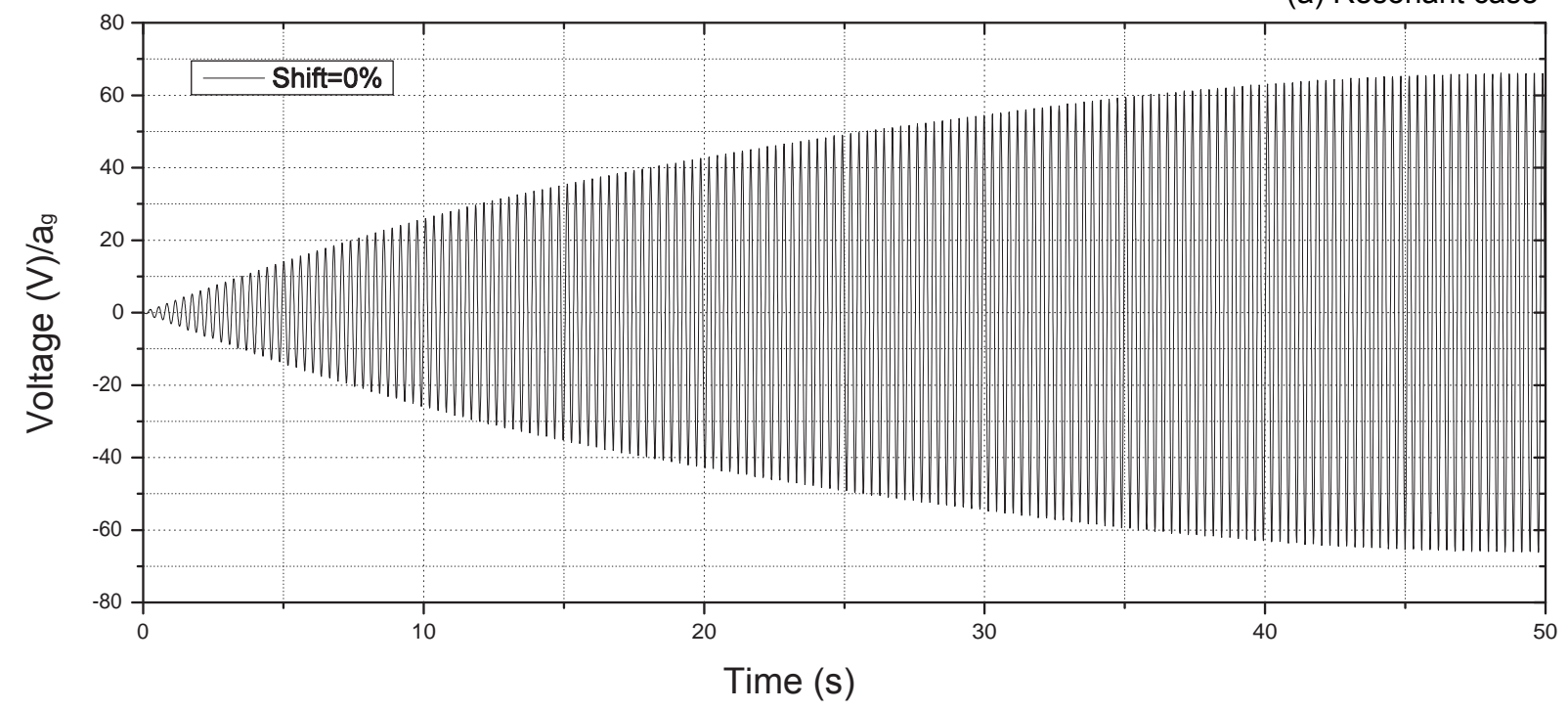

(b) Influence of shift in the resonant frequency

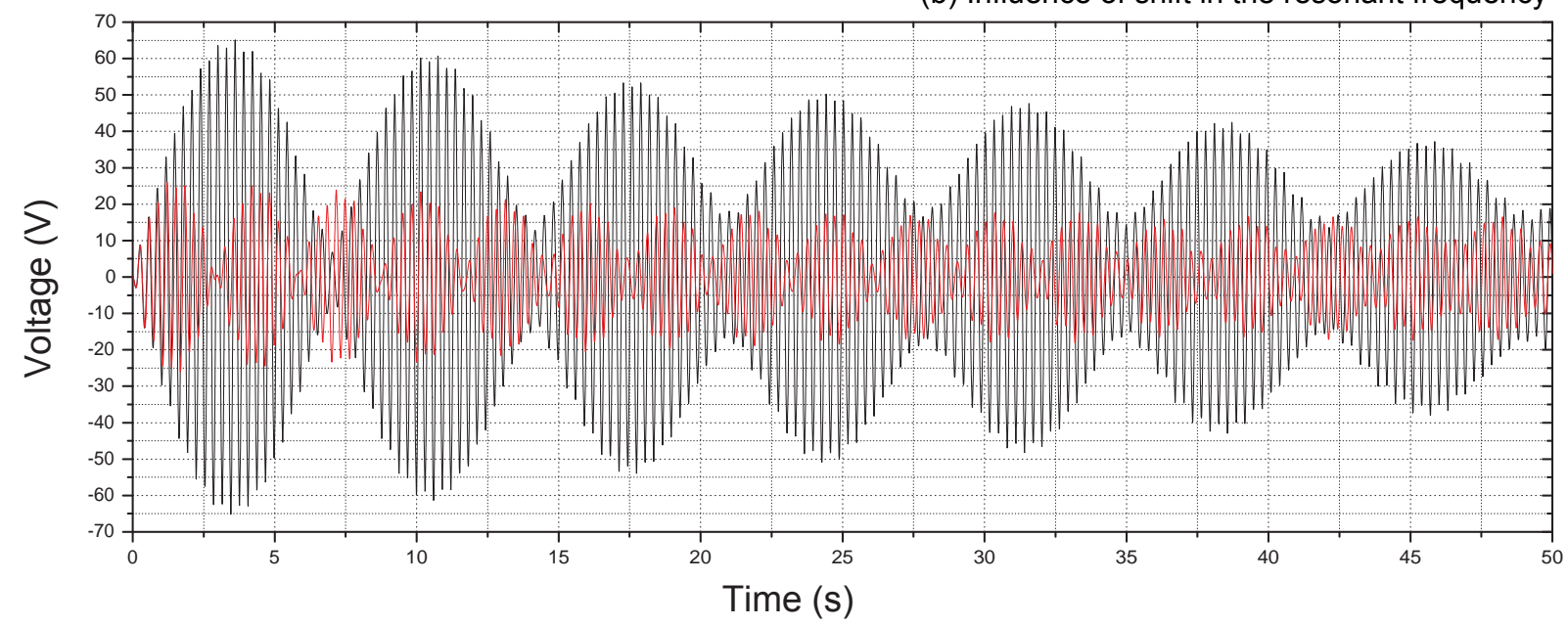

Figure 17: Analysis of the beat phenomenon (in case of frequency shift, the red line denotes a shift equal to $10 \%$ while the black line corresponds to a shift equal to $5 \%$ ). 

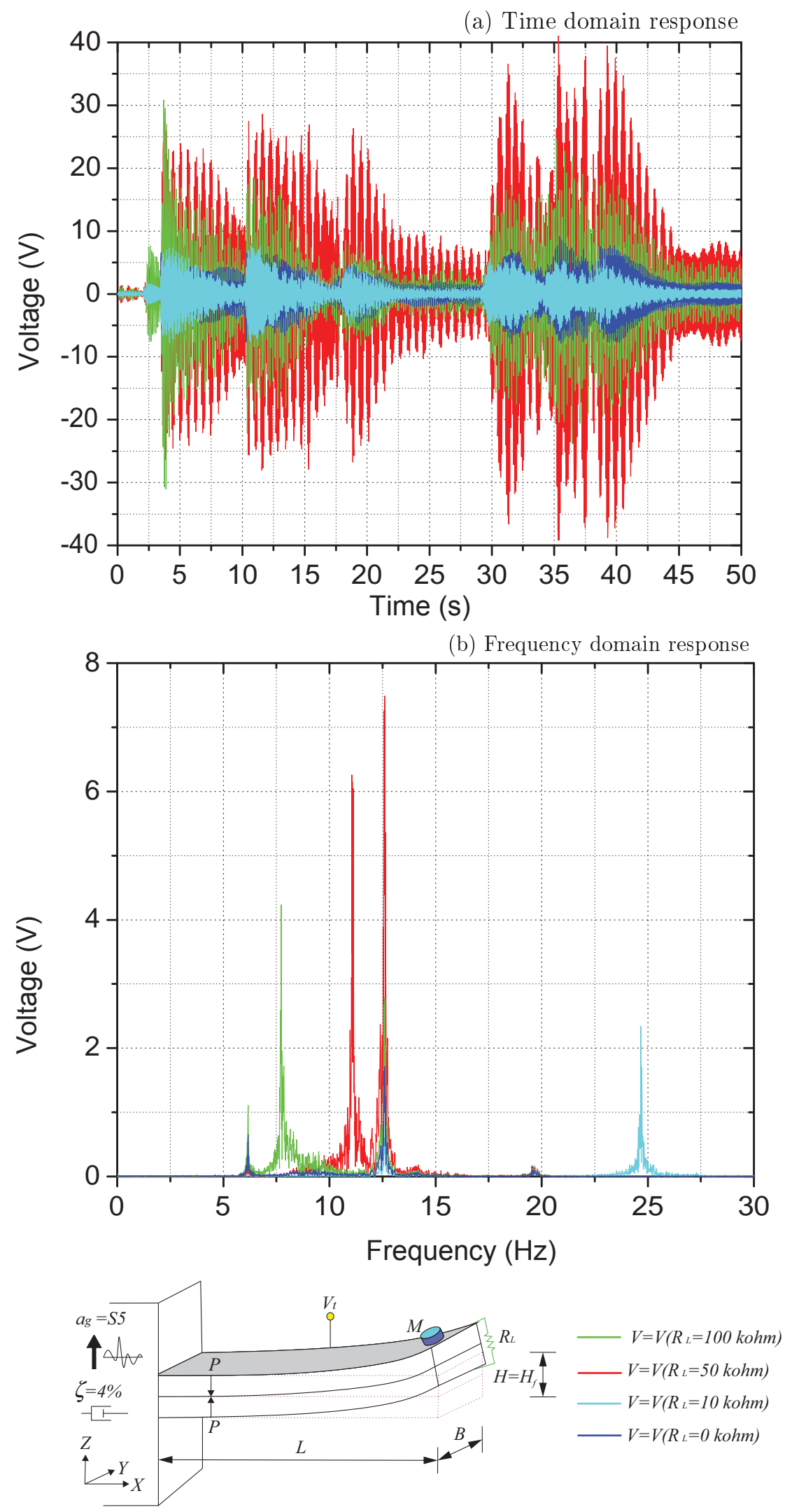

Figure 18: Sensitivity to electrical resistance. 
[11] Feng Ding, Xiaoping Peter Liu and Guangjun Liu (2011). Identification methods for Hammerstein nonlinear systems. Elsevier Science, Digital Signal Processing, Vol.21(2), pp. 215-238.

[12] Lennart Ljung (2010). Perspectives on system identification. Elsevier Science, Annual Reviews in Control, Vol.34(1), pp. 1-12.

[13] Pfeiffer, F., and Bremer, H., (2017). The Art of Modeling Mechanical Systems, Vol.570, The Art of Modeling in Solid Mechanics. CISM International Centre for Mechanical Sciences, Chapter 6, pp. 321-386.

[14] Chatzi Eleni and Papadimitriou Costas (2016). Identification Methods for Structural Health Monitoring, Vol.567, Implementation of Parametric Methods for the Treatment of Uncertainties in Online Identification. CISM International Centre for Mechanical Sciences, Chapter 3, pp. 5187.

[15] Heinz Unbehauen and Ganti Prasada Rao (1987). Identification of continuous systems, NorthHolland, Systems and control series, Elsevier Science, Amsterdam.

[16] Andrew P. Sage and James L. Melsa (1971). System Identification, Academic Press.

[17] Rolf Isermann and Marco Muenchhof (2011). Identification of Dynamic Systems: An Introduction with Applications, Springer-Verlag.

[18] Rik Pintelon and Johan Schoukens (2012). System Identification: A Frequency Domain Approach, North-Holland, Systems and control series, Elsevier Science, Amsterdam. Wiley.

[19] Malatkar P., Nayfeh A. H. (2003). A Parametric Identification Technique for Single-Degree-ofFreedom Weakly Nonlinear Systems with Cubic Nonlinearities. Journal of vibration and control, Vol.9(3-4), pp. 317-336.

[20] Elvin N.G., Lajnef N. and Elvin A. (2006). Feasibility of structural monitoring with vibration powered sensors. Smart Materials and Structures, Vol.15(4).

[21] Erturk A. and Inman D.J. (2009). An experimentally validated bimorph cantilever model for piezoelectric energy harvesting from base excitations. Smart Mater. Struct. 18, 025009, 18 pp.

[22] Erturk A. and Inman D.J. (2011). Parameter identification and optimization in piezoelectric energy harvesting: analytical relations, asymptotic analyses, and experimental validations. Proc. IMechE, Vol. 225, Part I: J. Systems and Control Engineering.

[23] Zhao S. and Erturk A. (2013). Electroelastic modeling and experimental validations of piezoelectric energy harvesting from broadband random vibrations of cantilevered bimorphs. Smart Materials and Structures 22 (2013): 015002.

[24] Stanton S.C., Erturk A., Mann B.P. and Inman D. J. (2010). Nonlinear piezoelectricity in electroelastic energy harvesters: Modeling and experimental identification. Journal of Applied Physics 108, 074903.

[25] Porfiri, M., Maurini, C., Pouget, J. (2007). Identification of electromechanical modal parameters of linear piezoelectric structures. Smart Materials and Structures, Vol.16(2), pp. 323-331.

[26] Delpero, T., Bergamini, A. E. and Ermanni, P. (2013). Identification of electromechanical parameters in piezoelectric shunt damping and loss factor prediction. Journal of Intelligent Material Systems and Structures, Vol.24(3), pp. 287-298. 
[27] Lavretsky, E. and Wise K. A. (2013). Robust and Adaptive Control With Aerospace Applications, Springer.

[28] Sergey Edward Lyshevski (2008). Electromechanical systems and devices, CRC Press.

[29] Xu Wang (2016). Frequency Analysis of Vibration Energy Harvesting Systems, Academic Press.

[30] Bompard, E., Ciwei, G., Napoli, R., Torelli, F. (2007). Dynamic price forecast in a competitive electricity market. IET Generation Transmission and Distribution, Vol.1(5).

[31] Torelli F. and Vaccaro A., Xie N. (2013). A Novel Optimal Power Flow Formulation Based on the Lyapunov Theory. IEEE Transactions on Power Systems, Vol.28(4), pp. 4405-4415.

[32] Bompard, E., Vaccaro, A., Xie, N., Torelli, F. (2013). Dynamic computing paradigm for comprehensive power flow analysis. The Institution of Engineering and Technology, IET Generation Transmission and Distribution, Vol.7(8), pp. 832-842.

[33] Torelli F. and Vaccaro A. (2014). A generalized computing paradigm based on artificial dynamic models for mathematical programming. Soft Computing, Vol. 18(8), pp. 1561-1573.

[34] Torelli F., Vaccaro A. (2015). A second order dynamic power flow model. Elsevier Science, Electric Power Systems Research, Vol.126, pp. 12-20.

[35] Novakovic Z.R. (1990). Solving systems of non-linear equations using the Lyapunov direct method. Elsevier Science, Computers and Mathematics with Applications, Vol.20(12), pp. 1923.

[36] Maruccio, C., Acciani, G., Montegiglio, P., Torelli, F. (2017). A novel computing paradigm for parameter identification of piezoelectric energy harvesting systems subjected to uncertain loads. Proceedings of the 9th European Conference on Offshore Wind and other marine renewable Energies in Mediterranean and European Seas (OWEMES17), Bary (Italy).

[37] Sehitoglu, H. (1983). Real-time parameter identification in a class of distributed systems using Lyapunov design method Parti. Theory. Taylor and Francis Group, International Journal of Control, Vol.38(4), pp. 747-756.

[38] Sehitoglu, H. (1983). Real-time parameter identification in a class of distributed systems using Lyapunov design method Part II. Applications. Taylor and Francis Group, International Journal of Control, Vol.38(4), pp. 757-767.

[39] Junhong Li, Ruifeng Ding, Yi Yang (2012). Iterative parameter identification methods for nonlinear functions. Elsevier Science, Applied Mathematical Modelling, Vol.366, pp. 2739-2750.

[40] Junhong Li (2013). Parameter estimation for Hammerstein CARARMA systems based on the Newton iteration. Elsevier Science, Applied Mathematics Letters, Vol.261.

[41] Dehghan Mehdi and Hajarian Masoud (2012). Fourth order variants of Newton's method without second derivatives for solving non linear equations. Emerald Group Publishing Limited, Engineering Computations, Vol.294, pp. 356-365.

[42] Giuseppe Quaranta, Giorgio Monti and Giuseppe C. Marano (2010). Parameters identification of Van der Pol - Duffing oscillators via particle swarm optimization and differential evolution. Mechanical Systems and Signal Processing, Vol. 247, pp. 2076-2095. 
[43] Giuseppe Quaranta, Giuseppe C. Marano, Rita Greco and Giorgio Monti (2014). Parametric identification of seismic isolators using differential evolution and particle swarm optimization. Applied Soft Computing, Vol. 22, pp. 458-464.

[44] Persano L., Dagdeviren C., Maruccio C., De Lorenzis L. and Pisignano D. (2014). Cooperativity in the enhanced piezoelectric response of polymer nanowires. Advanced Materials, 26, 757480.

[45] Maruccio C. and De Lorenzis L. (2014) Numerical homogenization of piezoelectric textiles for energy harvesting. Frattura ed Integritá Strutturale, 29, 4960.

[46] Maruccio C., De Lorenzis L., Persano L. and Pisignano D. (2015). Computational homogenization of fibrous piezoelectric materials. Computational Mechanics, 55, 98398.

[47] Maruccio C., Quaranta G., De Lorenzis L. and Monti G. (2016). Energy harvesting from electrospun piezoelectric nanofibers for structural health monitoring of a cable-stayed bridge. Smart Materials and Structures, 25(8), 085040.

[48] Quaranta G., Trentadue F., Maruccio C. and Marano G. C. (2018). Analysis of piezoelectric energy harvester under modulated and filtered white Gaussian noise. Mechanical Systems and Signal Processing, 104, 134-144.

[49] Maruccio C., Quaranta G., Montegiglio P., Trentadue F. and Acciani G. (2018). A Two Step Hybrid Approach for Modeling the Nonlinear Dynamic Response of Piezoelectric Energy Harvesters. Hindawi, Shock and Vibration, pp. 1-22.

[50] Landau, I. D. (1972). Model Reference Adaptive SystemsA Survey (MRAS)What is Possible and Why? Journal of Dynamic Systems Measurement and Control, Vol.94(2).

[51] Peter Young (1981). Parameter estimation for continuous time models - A survey. Elsevier Science, Automatica, Vol.17(1), pp. 23-39.

[52] Brufau-Penella, J., Tsiakmakis, K., Laopoulos, T., Puig-Vidal, M. (2008). Model reference adaptive control for an ionic polymer metal composite in underwater applications. Institute of Physics, Smart Materials and Structures, Vol.17(4).

[53] Torabi, K., Amiri Moghadam, A. A. (2012). Robust control of conjugated polymer actuators considering the spatio-temporal dynamics. Proc. of the Institution of Mechanical Engineers Part I: Journal of Systems and Control Engineering, Vol.226(6), pp. 806-822.

[54] McDaid, A.J., Haemmerle, E., Xie, S.Q. (2012). Control of IPMC Actuators for Microfluidics With Adaptive Online Iterative Feedback Tuning. IEEE/ASME Transactions on Mechatronics, Vol.17(4).

[55] McDaid, A. J., Haemmerle, E., Shahinpoor, M., Xie, S. Q. (2012). Adaptive tuning of a 2DOF controller for robust cell manipulation using IPMC actuators. Journal of Micromechanics and Microengineering, Vol.21(12).

[56] Bathe K. J. and Gracewski S. (1981). On nonlinear dynamic analysis using substructuring and mode superposition. Computers and Structures, 13(5), 699-707.

[57] Ansys-Coupled field analysis guide. ANSYS INC., Canonsburg (PA).

[58] Anil K. Chopra and Rakesh K. Goel (2002). A modal pushover analysis procedure for estimating seismic demands for buildings. Earthquake Engineering and Structural Dynamics. Vol.31(3), pp. $561-582$. 
[59] Adhikari S, Friswell MI and Inman DJ (2009) Piezoelectric energy harvesting from broadband random vibrations. Smart Materials and Structures 18: 115005.

[60] Arrieta AF, Delpero T, Bergamini AE and Ermanni P (2013) Broadband vibration energy harvesting based on cantilevered piezoelectric bi-stable composites. Applied Physics Letters 102: 173904.

[61] Bilgen O, Friswell MI, Faruque Ali S and Litak G (2015) Broadband vibration energy harvesting from a vertical cantilever piezocomposite beam with tip mass. International Journal of Structural Stability and Dynamics 15(2): 1450038.

[62] Dong S, Zhai J, Li J, Viehland D and Priya S (2008) Multimodal system for harvesting magnetic and mechanical energy. Applied Physics Letters 93: 103511.

[63] Dutoit NE and Wardle BL (2006) Performance of microfabricated piezoelectric vibration energy harvesters. Integrated Ferroelectrics 83: 13-32.

[64] Elvin NG and Elvin AA (2011) An experimentally validated electromagnetic energy harvester. Journal of Sound and Vibration 330: 2314-2324.

[65] Erturk A, Hoffmann J and Inman DJ (2009) A piezomagnetoelastic structure for broadband vibration energy harvesting. Applied Physics Letters 94: 254102.

[66] Erturk A and Inman DJ (2009) An experimentally validated bimorph cantilever model for piezoelectric energy harvesting from base excitations. Smart Materials and Structures 18: 025009 .

[67] Erturk A and Inman DJ (2011) Piezoelectric energy harvesting, Wiley, Chichester.

[68] Glynne-Jones P, Tudor MJ, Beeby SP and White NM (2004) An electromagnetic, vibrationpowered generator for intelligent sensor systems. Sensors and Actuators A: Physical 110: 344349.

[69] Hancke GP, Silva BC and Hancke Jr. GP (2013) The role of advanced sensing in smart cities. Sensors 13(1): 393-425.

[70] Lallart M, Anton SR and Inman DJ (2010) Frequency self-tuning scheme for broadband vibration energy harvesting. Journal of Intelligent Material Systems and Structures 21(9): 897-906.

[71] Mitcheson PD, Miao P, Stark BH, Yeatman E, Holmes A and Green T (2004) MEMS electrostatic micropower generator for low frequency operation. Sensors and Actuators A: Physical 115: 523-529.

[72] Muthalif AGA and Nordin NHD (2014) Optimal piezoelectric beam shape for single and broadband vibration energy harvesting: Modeling, simulation and experimental results. Mechanical Systems and Signal Processing 54-55: 417-426.

[73] Priya S and Inman DJ (2009) Energy harvesting technologies, Springer, New York.

[74] Roundy S and Wright PK (2004) A piezoelectric vibration based generator for wireless electronics. Smart Materials and Structures 13: 1131-1142.

[75] Tang L, Yang Y and Soh CK (2010) Toward broadband vibration-based energy harvesting. Journal of Intelligent Material Systems and Structures 21(18): 1867-1897 
[76] Tvedt LGW, Nguyen DS and Halvorsen E (2010) Nonlinear behavior of an electrostatic energy harvester under wide-and narrowband excitation. Journal of Microelectromechanical Systems 19: 305-316.

[77] Twiefel J and Westermann H (2913) Survey on broadband techniques for vibration energy harvesting. Journal of Intelligent Material Systems and Structures 24(11): 1291-1302.

[78] Wang L and Yuan F (2008) Vibration energy harvesting by magnetostrictive material. Smart Materials and Structures 17: 045009.

[79] Liu JQ, Fang HB and Xu ZY (2008) A MEMS-based piezoelectric power generator array for vibration energy harvesting. Microelectronics Journal 39: 802-806.

[80] Xue H, Hu Y andWang QM (2008) Broadband piezoelectric energy harvesting devices using multiple bimorphs with different operating frequencies. IEEE Transactions on Ultrasonics Ferroelectrics and Frequency Control 55: 2104-2108.

[81] Elvin NG, Lajnef N and Elvin AA (2006) Feasibility of structural monitoring with vibration powered sensors. Smart Materials and Structures 15(4): 977.

[82] Kaltenbacher M. (2015) Numerical Simulation of Mechatronic Sensors and Actuators. Springer.

[83] Clark V, Marepalli P and Bansal R (2012) Modeling, Design and Simulation of N/MEMS by Integrating Finite Element, Lumped Element and System Level Analyses. Chapter 3, Computational Finite Element Methods in Nanotechnology, CRC Press, ISBN 9781439893234. 41-84.

[84] Bathe KJ (1982) Finite Element Procedures in Engineering Analysis Prentice-Hall.

[85] IEEE Standard on Piezoelectricity, 1987. Vol. 176, ANSI/IEEE Std.

[86] Maruccio C, Quaranta G, De Lorenzis L and Monti G (2016) Energy harvesting from electrospun piezoelectric nanofibers for structural health monitoring of a cable-stayed bridge. Smart Materials and Structures 25:8.

[87] Maruccio C, Quaranta G, De Lorenzis L and Monti G (2016) Large deflection effects in flexible energy harvesters. Journal of Intelligent Material Systems and Structures 23:13. 1475-1484.

[88] Tang L, Yang Y and Kiong Soh C (2013) Broadband Vibration Energy Harvesting Techniques. Advances in Energy Harvesting Methods. Editors: Niell E, Erturk A. Springer.

[89] Blokhina E, El Aroudi E, Alarcon E, Galayko D. (2016) Nonlinearity in Energy Harvesting Systems: Micro- and Nanoscale Applications. Springer.

[90] Sun C, Shi J, Bayerl D and Wang X. (2011) PVDF microbelts for harvesting energy from respiration. Energy Environ. Sci.. 4: 4508.

[91] Cahill P, Hazra B, Karoumi R, Mathewson A and Pakrashi V. (2018) Vibration energy harvesting based monitoring of an operational bridge undergoing forced vibration and train passage. Mechanical Systems and Signal Processing. 106: 265-283.

[92] Cahill P, Mathewson A and Pakrashi V. (2018) Experimental Validation of Piezoelectric Energy Harvesting Device for Built Infrastructure Application. ASCE Journal of Bridge Engineering. 23(8): 04018056-1-11. 
[93] Sazonov E, Curry D, Pillay P. (2009) Self-powered sensors for monitoring of highway bridges. IEEE Sens. J. 9(11): 1422-1429.

[94] Peigney M, Siegert D (2013) Piezoelectric energy harvesting from traffic induced bridge vibrations. Smart Mater. Struct. 22(9): 095019. 